\title{
Symmetry algebras of stringy cosets
}

\section{${\text { Dushyant } \mathrm{Kumar}^{a} \text { and Menika Sharma }}^{b}$}

\author{
${ }^{a}$ Harish-Chandra Research Institute, \\ Jhusi, Allahabad, India \\ ${ }^{b}$ Department of Mathematics, City, University of London, \\ Northampton Square, EC1V 0HB London, U.K. \\ E-mail: sehrawat.dushyant@gmail.com, menika.sharma@city.ac.uk
}

ABSTRACT: We find the symmetry algebras of cosets which are generalizations of the minimal-model cosets, of the specific form $\frac{\mathrm{SU}(N)_{k} \times \mathrm{SU}(N)_{\ell}}{\mathrm{SU}(N)_{k+\ell}}$. We study this coset in its free field limit, with $k, \ell \rightarrow \infty$, where it reduces to a theory of free bosons. We show that, in this limit and at large $N$, the algebra $\mathcal{W}_{\infty}^{e}[1]$ emerges as a sub-algebra of the coset algebra. The full coset algebra is a larger algebra than conventional $\mathcal{W}$-algebras, with the number of generators rising exponentially with the spin, characteristic of a stringy growth of states. We compare the coset algebra to the symmetry algebra of the large $N$ symmetric product orbifold CFT, which is known to have a stringy symmetry algebra labelled the 'higher spin square'. We propose that the higher spin square is a sub-algebra of the symmetry algebra of our stringy coset.

Keywords: Conformal and W Symmetry, Higher Spin Symmetry, AdS-CFT Correspondence

ARXIV EPRINT: 1812.11920 


\section{Contents}

1 Introduction 1

2 Perturbative growth of states 3

2.1 Algebra for small $N \quad 5$

2.1.1 Algebra for stringy SU(2) coset 5

2.1.2 Algebra for stringy SU(3) coset 6

$\begin{array}{ll}2.2 & \text { Algebra for cosets with finite } k\end{array}$

2.3 Asymptotic growth of vacuum character 8

3 Generalized Casimir current algebra $\quad 9$

3.1 General $k$ and $k=1 \quad 10$

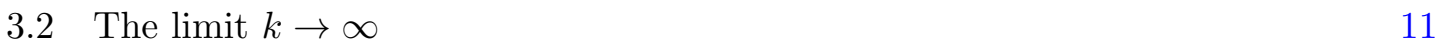

$\begin{array}{lll}3.2 .1 \quad N=2 & 12\end{array}$

$\begin{array}{lll}3.2 .2 \quad N=3 & 14\end{array}$

$\begin{array}{lll}3.2 .3 & \text { Large } N & 17\end{array}$

$\begin{array}{lll}3.3 & \text { Relation with } \mathcal{W}_{\infty}^{e}[1] \text { and the higher spin square } & 19\end{array}$

$\begin{array}{lll}4 & \text { Discussion } & 21\end{array}$

$\begin{array}{lr}\text { A The } \mathrm{SU}(N) \text { tensor invariants } & 23\end{array}$

$\begin{array}{ll}\text { B Primary fields } & 24\end{array}$

$\begin{array}{ll}\text { C Algebra of symmetric product orbifold CFT } & 24\end{array}$

\section{Introduction}

The papers [1-3] established a duality between the CFT of the coset model

$$
\frac{\mathrm{SU}(N)_{1} \times \mathrm{SU}(N)_{\ell}}{\mathrm{SU}(N)_{1+\ell}}
$$

and three-dimensional higher-spin Vasiliev theory [4-6], in the large $N, \ell$ limit. This duality is characterized by a large symmetry algebra $W_{\infty}[\mu]$ on the CFT side which is interpreted as the asymptotic symmetry algebra on the bulk side [7-9]. The parameter $\mu=\frac{N}{\ell+N}$ is the 't Hooft coupling on the CFT side, while on the bulk side it determines the mass of the scalar field. The $\mathcal{W}_{\infty}[\mu]$ algebra consists of generators with spin 2 to $\infty$ with each generator having multiplicity one and the commutation relations of these generators depend on the parameter $\mu$.

Our aim is to find the symmetry algebra for the coset

$$
\frac{\mathrm{SU}(N)_{k} \times \mathrm{SU}(N)_{\ell}}{\mathrm{SU}(N)_{k+\ell}}
$$


which is a generalization of the coset in eq. (1.1). The central charge for this coset is

$$
\frac{k \ell(k+\ell+2 N)\left(N^{2}-1\right)}{(k+N)(\ell+N)(k+\ell+N)} .
$$

The coset in eq. (1.2) has three independent parameters and two interesting limits. The first limit arises on taking $N$ and $\ell$ to infinity while holding $k$ and $\mu=\frac{N}{N+\ell}$ fixed. The central charge reduces to

$$
\frac{k\left(N^{2}-1\right)}{k+N} \sim k N\left(1-\mu^{2}\right)
$$

Since, the central charge scales as $\sim N$, in this limit the coset in eq. (1.2) is usually referred to as a vector coset model. This vector coset model was studied in refs. [10, 11], where a related, but different, coset model $\mathrm{SU}(k+\ell)_{N} / \mathrm{SU}(\ell)_{N}$ was proposed as the CFT dual to a bulk Vasiliev theory with a matrix extension.

There exist other limiting procedures which result in a central charge of the coset in eq. (1.2) which scales as $N^{2}$. One way is to take $N$ and $k, \ell$ to infinity while holding $k-\ell$ and $\mu=\frac{N}{k+\ell+N}$ fixed. In this case, the central charge scales as $\sim N^{2}\left(1-\frac{1}{\mu}\right)$. A variation of this, which is the limiting procedure we use in this paper, is to take $N$ and $k, \ell$ to infinity with $N / \ell$ set to zero and

$$
\lambda=\frac{N}{k}
$$

fixed. The coset central charge is then

$$
\frac{N^{2}-1}{1+\lambda}
$$

Since the central charge scales as a matrix model, in this limit we expect the coset in eq. (1.2) to have a string dual and we refer to it in the text as the stringy $\mathrm{SU}(N)$ coset. In this paper, we will study the symmetry algebra of this stringy $\mathrm{SU}(N)$ coset in the limit where $k$ and $\ell$ go to infinity, but keep $N$ finite. Thus, we are not determining the algebra explicitly at large $N$. However, from the general behavior of the algebra at finite $N$, we can infer many of its properties at infinite $N$ which we will elaborate on in the text. In particular, this method tells us the properties of the large $N$ coset algebra at $\lambda=0$ with $\lambda$ defined as in eq. (1.5).

Historically, the algebra for the coset model in eq. (1.1) was first studied at finite $N$ before the infinite $N$ case was dealt with. The finite $N$ algebra is called $\mathcal{W}_{N}$ [12] and has generators ranging from 2 to $N$ with multiplicity one. Indeed, it has taken many years to completely understand the large $N$ limit of the $\mathcal{W}_{N}$ algebra [13-15]. The algebra of the coset in eq. (1.2) for small $N$ and large $k, \ell$ has been studied before in refs. [16-18], although it has only attracted a fraction of the attention that the $\mathcal{W}_{N}$ algebra has and perhaps rightly so. $\mathcal{W}_{N}$ algebras, which are extensions of the Virasoro algebra, have complicated commutation relations but a simple spectrum of fields. In contrast, the symmetry algebras of the coset theories in eq. (1.2) have a spectrum of generators with the multiplicity climbing at a exponential rate with the spin (the algebra still has, of course, a finite number of generators at finite $N$ ). Unlike their $\mathcal{W}_{N}$ counterparts, these algebras belong to a class of algebras 
which are finitely non-freely generated [19] and thus are less tractable. On the other hand, the fact that this coset model and supersymmetric generalizations have generators whose multiplicity increases with spin makes them prime candidates to be dual to string theories in AdS. It is with this motivation that we study them in this paper.

We exclusively work with the bosonic coset in eq. (1.2), so that we can study the symmetry algebra in its simplest form. A $\mathcal{N}=2$ supersymmetric generalization of the coset in eq. (1.2) was studied in [20]. Related work for a coset with $\mathcal{N}=1$ supersymmetry appears in refs. [21-24]. However, a crucial distinction between our analysis and the supersymmetric cases studied is that we are working in the limit of zero coupling, with a free theory.

The coset theories that we study are similar to $\mathrm{SU}(N)$ gauge theories in four dimensions which are known to have string duals on the $A d S_{5}$ background. However, string theories on $A d S_{3}$ are expected to be dual to a different family of CFTs: symmetric product orbifolds. In this paper, we explore the relation between the symmetry algebra of the bosonic symmetric product orbifold theory and the coset theory. To be able to do this, we explicitly write down the currents of the coset theory. For the coset in eq. (1.1) with level $k=1$, the currents of the $\mathcal{W}$-algebra correspond to Casimir operators of $\mathrm{SU}(N)$. For the more general coset theory, currents of the $\mathcal{W}$-algebra can be generated from the Casimir operators by sprinkling additional derivatives on the constituent currents. We construct these currents in section 3. However, as we will see, the coset theory also has additional currents that cannot be constructed from the Casimir operators.

Information about the generators of any coset theory resides in the vacuum character of the partition function of the theory. In section 2, we write down the vacuum character of the coset in eq. (1.2). Unlike the case $k=1$, it is not possible to formulate this character in closed form for general $k$ and $N$ and it can only be expressed in terms of string functions. We therefore resort to numerical techniques to find the generators of the algebra from its vacuum character for low values of $N$, following ref. [16]. Later in section 3, when we explicitly construct the currents for finite $N$, the calculation in section 2 serves as a touchstone for our results.

This paper is organized as follows. In section 2 we find the low lying spectrum of the symmetry algebra of the coset in eq. (1.2) for small values of $N$ in the large $k, \ell$ limit. In section 3 we construct the currents for this same coset for the special values $N=2$ and $N=3$. The $N=3$ case is especially important for understanding the structure of the currents at general $N$ and we present this case in some detail. In section 3, we also work out the relation of the coset algebra to the algebra $\mathcal{W}_{\infty}[\mu]$ and also its relation to the higher spin square. The algebra of the symmetric product orbifold at general values of $N$ is worked out in appendix C.

\section{Perturbative growth of states}

In this section, we compute the vacuum character of the coset model in eq. (1.2) at finite $N$, with $k, \ell \rightarrow \infty$. This computation will tell us at what rate the perturbative states of the current algebra grow with the spin. The density of states of a CFT partition function in the regime of large spin $s$ but $s<c$ determines the dual holographic theory. Since, in 
this paper, we are only interested in the symmetry algebra of the coset in eq. (1.2), we will focus on the vacuum character. We will not be able to determine the vacuum character exactly but will compute the low-lying spectrum of the symmetry algebra. We carry this out in section 2.1 and our results appear in table 1. It is also of interest to compute the vacuum character at fixed $k$ at finite $N$, since this helps us to understand the nature of the symmetry algebra of our stringy coset. We do this in section 2.2 and the results appear in tables 2 and 3. In section 2.3, we determine the asymptotic growth of states of the vacuum character. In the following, we describe the method we use to compute the vacuum character.

It is well known that the $\mathcal{W}$-algebra of the coset $\mathfrak{g}_{k} / \mathfrak{g}$ is the same as the $\mathcal{W}$-algebra of the coset $\left(\mathfrak{g}_{k} \oplus \mathfrak{g}_{\ell}\right) / \mathfrak{g}_{k+\ell}$ in the $\ell \rightarrow \infty$ limit $[16,25]$. Therefore, to find the symmetry algebra for the coset

$$
\frac{\mathrm{SU}(N)_{k} \otimes \mathrm{SU}(N)_{\ell}}{\mathrm{SU}(N)_{k+\ell}}
$$

as $\ell \rightarrow \infty$, we find the algebra of the coset model

$$
\frac{\mathrm{SU}(N)_{k}}{\mathrm{SU}(N)}
$$

using its vacuum character.

The characters of the coset in eq. (2.2), also known as branching functions, depend on the weights $\mathrm{SU}(N)_{k}$ and $\mathrm{SU}(N)$, which we label by $\Lambda$ and $\lambda$ respectively. The branching function for the coset is given by $[16,26]$

$$
b_{\lambda}^{\Lambda}(q) \equiv \operatorname{Tr}_{L_{\Lambda, \lambda}} q^{L_{0}-c / 24}=\sum_{w \in W} \epsilon(w) c_{w(\lambda+\rho)-\rho+k \Lambda_{0}}^{\Lambda}(q) q^{\frac{1}{2 k}|w(\lambda+\rho)-\rho|^{2}} .
$$

The sum on the r.h.s. is over the group elements $w$ of the Weyl group $W$ of $\mathrm{SU}(N)$, which is $S_{N}$. The determinant of $w$ is labelled as $\epsilon(w)$ and takes values: $\pm 1 . \rho$ is the Weyl vector, equal to half the sum of the positive roots of $\mathrm{SU}(N) . \Lambda_{0}$ is the vacuum weight of $\mathrm{SU}(N)_{k}$ given by $(k, 0, \cdots)$. The $c_{\lambda}^{\Lambda}(q)$ are the Kac-Peterson string functions defined in eq. (2.7).

To find the coset symmetry algebra we need to look at the vacuum character, so we work out the branching function $b_{\lambda}^{\Lambda}(q)$ for the weights: $\Lambda=(k, 0, \cdots)$ and $\lambda=(0,0, \cdots)$. This will give us a series in the variable $q$. We can rearrange this series as

$$
\frac{q^{-\frac{c}{24}}}{\prod_{i=1}^{l} F_{s_{i}}(q)}\left(1-j q^{n} \cdots\right),
$$

where

$$
F_{s} \equiv \prod_{k=s}^{\infty}\left(1-q^{k}\right) .
$$

eq. (2.4) is the general form of the vacuum character for an algebra with fields of spins $s_{i}$, where $i$ ranges from 1 to $l$ and with $j$ null states starting at order $n$. Here, $l$ is the total number of generators of the algebra. This is the so-called "character technique". Note that this technique is not fool-proof. The actual algebra may have additional currents, since we can always add currents to the denominator of eq. (2.4), while at the same time 
increasing the number of null states to keep the vacuum character unchanged. Nevertheless, studying the vacuum character gives us a good indication of the nature of the algebra. We also restrict our attention to the vacuum character and ignore any extensions of the coset algebra at specific values of the level $k$.

\subsection{Algebra for small $N$}

We now take $k$ to $\infty$. Then the branching function in eq. (2.3) reduces to

$$
b_{\lambda}^{\Lambda}(q)=\sum_{w \in W} \epsilon(w) c_{w(\lambda+\rho)-\rho+k \Lambda_{0}}^{\Lambda}(q) .
$$

The string functions for any affine group $\mathfrak{g}_{k}$ are given by

$$
c_{\lambda}^{\Lambda}(q)=\frac{q^{-c / 24}}{\prod\left(1-q^{n}\right)^{\operatorname{dim} \mathbf{g}}} \sum_{w \in \widehat{W}} \epsilon(w) q^{h_{w * \Lambda, \lambda}} \sum_{\left\{n_{\beta} \in \mathbb{Z} \mid \sum_{\beta \in \Delta_{+}} n_{\beta} \beta=w * \Lambda-\lambda\right\}}\left(\prod_{\beta \in \Delta_{+}} \phi_{n_{\beta}}(q)\right),
$$

Here, $\widehat{W}$ is the affine Weyl group, while $\Delta_{+}$denotes the set of positive roots of $\mathfrak{g}$. We have also introduced

$$
\begin{aligned}
\phi_{n} & =\sum_{m \geq 0}(-1)^{m} q^{\frac{1}{2} m(m+1)+n m}, \quad \phi_{-n}(q)=q^{n} \phi_{n}(q), \\
h_{\Lambda, \lambda} & =\frac{(\Lambda, \Lambda+2 \rho)}{2\left(k+h^{V}\right)}-\frac{(\lambda, \lambda)}{2 k},
\end{aligned}
$$

and $w * \Lambda=w(\Lambda+\rho)-\rho$, while $h^{V}$ is the dual coexter number. In the large $k$ limit, the sum in eq. (2.7) over the affine Weyl group elements will reduce to a sum over the finite Weyl group elements. Thus the expression in eq. (2.7) simplifies to

$$
c_{\lambda}^{\Lambda}(q)=\frac{q^{-c / 24}}{\prod\left(1-q^{n}\right)^{\operatorname{dim} g}} \sum_{w \in W} \epsilon(w) \sum_{\left\{n_{\beta} \in \mathbb{Z} \mid \sum_{\beta \in \Delta_{+}} n_{\beta} \beta=w * \Lambda-\lambda\right\}}\left(\prod_{\beta \in \Delta_{+}} \phi_{n_{\beta}}(q)\right) .
$$

\subsubsection{Algebra for stringy $\mathrm{SU}(2)$ coset}

In this case, there are two Weyl group elements: 1 and $w_{\alpha_{1}}$. Thus, the branching function in eq. (2.6) becomes

$$
b_{(0)}^{(k, 0)}(q)=c_{(k, 0)}^{(k, 0)}(q)-c_{(k-2,2)}^{(k, 0)}(q) .
$$

In the limit $k \rightarrow \infty$, the string functions appearing in the r.h.s. of the above expression are given by

$$
c_{(k, 0)}^{(k, 0)}(q)=\frac{q^{-c / 24}}{\prod\left(1-q^{n}\right)^{3}}\left\{\phi_{0}(q)-\phi_{-1}(q)\right\}
$$

and

$$
c_{(k-2,2)}^{(k, 0)}(q)=\frac{q^{-c / 24}}{\prod\left(1-q^{n}\right)^{3}}\left\{\phi_{-1}(q)-\phi_{-2}(q)\right\} .
$$

These expressions can be read off from eq. (2.10). Working out (and rearranging) the branching function we get

$$
b_{(0)}^{(k, 0)}(q)=\frac{1}{F_{2} F_{4} F_{6}^{2} F_{8}^{2} F_{9} F_{10}^{2} F_{12}}\left(1-q^{13}-3 q^{14}-7 q^{15}-\cdots\right)
$$


Thus the coset $\frac{\mathrm{SU}(2)_{k}}{\mathrm{SU}(2)}$ has a symmetry algebra with generators of spin

$$
2,4,6^{2}, 8^{2}, 9,10^{2}, 12
$$

in the large $k$ limit.

\subsubsection{Algebra for stringy $\mathrm{SU}(3)$ coset}

The branching function in eq. (2.6) for the case of the coset $\frac{\mathrm{SU}(3)_{k}}{\mathrm{SU}(3)}$ becomes

$$
b_{(0,0)}^{(k, 0,0)}(q)=c_{(k, 0,0)}^{(k, 0,0)}(q)-2 c_{(k-2,1,1)}^{(k, 0,0)}(q)+c_{(k-3,3,0)}^{(k, 0,0)}(q)+c_{(k-3,0,3)}^{(k, 0,0)}(q)-c_{(k-4,2,2)}^{(k, 0,0)}(q) .
$$

Defining

$$
\zeta(q)=q^{-c / 24} \frac{1}{\prod\left(1-q^{n}\right)^{8}}
$$

and calculating the string functions in the limit $k \rightarrow \infty$ we find

$$
\begin{gathered}
c_{(k, 0,0)}^{(k, 0,0)}(q)=\zeta(q) \sum_{n \in \mathbb{Z}} \phi_{-n}(q)\left\{2 \phi_{n-1}(q) \phi_{n-2}(q)-2 \phi_{n-1}(q) \phi_{n}(q)\right. \\
\left.-\phi_{n-2}(q)^{2}+\phi_{n}(q)^{2}\right\} \\
c_{(k-2,1,1)}^{(k, 0,0)}(q) \zeta(q) \sum_{n \in \mathbb{Z}} \phi_{-n}(q)\left\{2 \phi_{n-2}(q) \phi_{n-3}(q)-2 \phi_{n-1}(q) \phi_{n-2}(q)\right. \\
\left.-\phi_{n-3}(q)^{2}+\phi_{n-1}(q)^{2}\right\} \\
c_{(k-3,3,0)}^{(k, 0,0)}(q)=\zeta(q) \sum_{n \in \mathbb{Z}} \phi_{-n}(q)\left\{\phi_{n-4}(q) \phi_{n-2}(q)-\phi_{n-4}(q) \phi_{n-3}(q)-\phi_{n-3}(q) \phi_{n-1}(q)\right. \\
\left.+\phi_{n-3}(q)^{2}+\phi_{n-2}(q) \phi_{n-1}(q)-\phi_{n-2}(q)^{2}\right\} \\
c_{(k-4,2,2)}^{(k, 0,0)}(q)=\zeta(q) \sum_{n \in \mathbb{Z}} \phi_{-n}(q)\left\{2 \phi_{n-3}(q) \phi_{n-4}(q)-2 \phi_{n-2}(q) \phi_{n-3}(q)\right. \\
\left.-\phi_{n-4}(q)^{2}+\phi_{n-2}(q)^{2}\right\} .
\end{gathered}
$$

In addition,

$$
c_{(k-3,0,3)}^{(k, 0,0)}(q)=c_{(k-3,3,0)}^{(k, 0,0)}(q) .
$$

Working out the branching function we get

$$
\begin{aligned}
b_{(0)}^{(k, 0)}(q)=\frac{1}{F_{2} F_{3} F_{4} F_{5} F_{6}^{4} F_{7}^{2} F_{8}^{7} F_{9}^{9} F_{10}^{12} F_{11}^{16} F_{12}^{26} F_{13}^{26} F_{14}^{33} F_{15}^{33} F_{16}^{12}} \\
\\
\times\left(1-24 q^{17}-137 q^{18}-404 q^{19}-\cdots\right) .
\end{aligned}
$$

The spin of the generators of the algebra and their multiplicity can now be read off from the denominator. Our results for the $N=2$ and $N=3$ cases agree with those in ref. [16].

We can work out the vacuum character for the coset $\frac{\mathrm{SU}(4)_{k}}{\mathrm{SU}(4)}$, in a similar fashion, and we find that the algebra has generators of spin:

$$
2,3,4^{2}, 5,6^{5}, 7^{4}, 8^{12}, 9^{15}, 10^{28}, 11^{41}, 12^{75}, 13^{103}, 14^{166}, 15^{235}, 16^{313}, 17^{362}, 18^{310} .
$$

Using Mathematica, we have worked out the symmetry algebra for the coset $\frac{\mathrm{SU}(N)_{k}}{\mathrm{SU}(N)}$ in the large $k$ limit till $N=7$ and up to generators of spin 8 . The results appear in table 1 . 


\begin{tabular}{|lll|}
\hline$N$ & Vacuum Character & Algebra \\
\hline 2 & $1+q^{2}+q^{3}+3 q^{4}+3 q^{5}+8 q^{6}+9 q^{7}+19 q^{8}+\cdots$ & $2,4,6^{2}, 8^{2}, \cdots$ \\
\hline 3 & $1+q^{2}+2 q^{3}+4 q^{4}+6 q^{5}+15 q^{6}+22 q^{7}+46 q^{8}+\cdots$ & $2,3,4,5,6^{4}, 7^{2}, 8^{7}, \cdots$ \\
\hline 4 & $1+q^{2}+2 q^{3}+5 q^{4}+7 q^{5}+18 q^{6}+29 q^{7}+64 q^{8}+\cdots$ & $2,3,4^{2}, 5,6^{5}, 7^{4}, 8^{12}, \cdots$ \\
\hline 5 & $1+q^{2}+2 q^{3}+5 q^{4}+8 q^{5}+19 q^{6}+32 q^{7}+71 q^{8}+\cdots$ & $2,3,4^{2}, 5^{2}, 6^{5}, 7^{5}, 8^{14}, \cdots$ \\
\hline 6 & $1+q^{2}+2 q^{3}+5 q^{4}+8 q^{5}+20 q^{6}+33 q^{7}+74 q^{8}+\cdots$ & $2,3,4^{2}, 5^{2}, 6^{6}, 7^{5}, 8^{15}, \cdots$ \\
\hline 7 & $1+q^{2}+2 q^{3}+5 q^{4}+8 q^{5}+20 q^{6}+34 q^{7}+75 q^{8}+\cdots$ & $2,3,4^{2}, 5^{2}, 6^{6}, 7^{6}, 8^{15}, \cdots$ \\
\hline
\end{tabular}

Table 1. The vacuum character for the stringy coset model for small values of $N$. The corresponding algebra appears in the third column. The central charge of the coset is related to $N$ by $c=N^{2}-1$. Note that the vacuum character (and hence the algebra) stabilizes till order $q^{N}$ : which means that the generators up to spin $N$ do not change on further increasing $N$.

As we can see from the table, the currents up to spin $N$ stop changing as $N$ is further increased. We, therefore, expect the algebra at $N=\infty$ to have the following low-lying spectrum of generators:

$$
2,3,4^{2}, 5^{2}, 6^{6}, 7^{6}, \cdots
$$

\subsection{Algebra for cosets with finite $k$}

To better understand the coset algebra in the infinite $k, \ell$ limit, it is instructive to find the algebra of the coset when $\ell$ is large but $k$ is fixed to a given value. The vacuum character for such a coset is given by eq. (2.3) and the string functions continue to be given by eq. (2.7). The string functions for a fixed level can be calculated in Mathematica using the package affine.m [27].

In table 2 we list the algebra for various $N$ for $k=3$. As can be seen, even for this low value of the level, the number of generators of the algebra grow quickly with the spin. In fact, for a fixed value of $N$, the coset algebra stabilizes for a small value of the level $k$ - that is the coset generators do not change after a certain level. This fact was earlier reported in [28]. As we show in table 3 , for $N=3$ the algebra has stabilized at level 8 . The field content at this level is identical to the field content at level $k=\infty$ calculated in eq. (2.20). Note that the number of null states continue to change and specifically decrease as we increase the level to infinity. The null states, however, never disappear from the spectrum and are present even in the infinite level limit, as can be see from the vacuum character in eq. (2.20).

Note that the growth rate of currents at finite $k$ is sharper than what might expect from the $T$-dual coset $\mathrm{SU}(k+\ell)_{N} /\left(\mathrm{SU}(k)_{N} \times \mathrm{SU}(\ell)_{N}\right)$. The symmetry algebra of this dual coset is expected to be a (truncation of) the matrix extension of $\mathcal{W}_{N}$ in the large $\ell, N$ limit for fixed $k$ - so the multiplicity for a given spin would be at most $k^{2}$. However, it is possible for dual cosets to have different symmetry algebras [25]. 


\begin{tabular}{|lll|}
\hline$N$ & Vacuum Character & Algebra \\
\hline 2 & $1+q^{2}+q^{3}+3 q^{4}+3 q^{5}+8 q^{6}+9 q^{7}+2,4,6^{2}, 8,9$ \\
& $19 q^{8}+25 q^{9}+45 q^{10}+61 q^{11}+105 q^{12}+$ \\
& $\cdots$ \\
\hline 3 & $1+q^{2}+2 q^{3}+4 q^{4}+6 q^{5}+15 q^{6}+2,3,4,5,6^{4}, 7^{2}, 8^{6}, 9^{8}, 10^{8}, 11^{10}, 12^{9}, \cdots$ \\
& $22 q^{7}+45 q^{8}+78 q^{9}+140 q^{10}+238 q^{11}+$ \\
& $426 q^{12}+\cdots$ \\
\hline 4 & $1+q^{2}+2 q^{3}+5 q^{4}+7 q^{5}+18 q^{6}+2,3,4^{2}, 5,6^{5}, 7^{4}, 8^{11}, 9^{14}, 10^{24}, 11^{34}, 12^{55}, \cdots$ \\
& $29 q^{7}+63 q^{8}+112 q^{9}+221 q^{10}+400 q^{11}+$ \\
& $771 q^{12}+\cdots$ \\
\hline 5 & $1+q^{2}+2 q^{3}+5 q^{4}+8 q^{5}+19 q^{6}+2,3,4^{2}, 5^{2}, 6^{5}, 7^{5}, 8^{13}, 9^{19}, 10^{34}, 11^{52}, 12^{94}, \cdots$ \\
& $32 q^{7}+70 q^{8}+130 q^{9}+261 q^{10}+490 q^{11}+$ \\
& $969 q^{12}+\cdots$ \\
\hline 6 & $1+q^{2}+2 q^{3}+5 q^{4}+8 q^{5}+20 q^{6}+2,3,4^{2}, 5^{2}, 6^{6}, 7^{5}, 8^{14}, 9^{21}, 10^{39}, 11^{62}, 12^{117}, \cdots$ \\
& $33 q^{7}+73 q^{8}+137 q^{9}+279 q^{10}+530 q^{11}+$ \\
& $1066 q^{12}+\cdots$ \\
\hline 7 & $1+q^{2}+2 q^{3}+5 q^{4}+8 q^{5}+20 q^{6}+2,3,4^{2}, 5^{2}, 6^{6}, 7^{6}, 8^{14}, 9^{22}, 10^{41}, 11^{67}, 12^{127}, \cdots$ \\
& $34 q^{7}+74 q^{8}+140 q^{9}+286 q^{10}+548 q^{11}+$ \\
& $1106 q^{12}+\cdots$ \\
\hline
\end{tabular}

Table 2. The vacuum character for the vector coset model for fixed $k=3$ and small values of $N$.

\subsection{Asymptotic growth of vacuum character}

The asymptotic growth of the vacuum character can be determined from the general formula for the asymptotic behaviour of branching functions [29]. Let us write the general branching function as

$$
b_{\lambda}^{\Lambda}(q)=\sum_{s} a_{s} q^{s}
$$

Then, asymptotically as $s \rightarrow \infty$,

$$
a_{s} \sim \frac{1}{2}(c / 6)^{1 / 4} b(\Lambda, \lambda) s^{-\frac{3}{4}} \exp (\pi \sqrt{2 / 3 c s})
$$

where, $b(\Lambda, \lambda)$ is a positive real number and $c$ is the central charge. Thus, in our case:

$$
a_{s} \sim s^{-\frac{3}{4}} \sqrt{N} \exp \left(\pi \sqrt{\frac{2}{3} N^{2} s}\right)
$$

where we have dropped the constants.

In spite of the fact that there is an exponential increase in the number of currents, for small spin $s$, a large number of null states occur as $s$ becomes greater than $N^{2}$ for the vacuum branching function, as we saw in the previous section. Hence, the vacuum character has Cardy growth at large $s$. Note that this asymptotic behaviour holds only for finite $N$ and may change for infinite $N$. 


\begin{tabular}{|llrl|}
\hline$k$ & Algebra & $k$ & Algebra \\
\hline 1 & $2,3$. & 5 & $2,3,4,5,6^{4}, 7^{2}, 8^{7}, 9^{9}, 10^{12}, 11^{16}, 12^{25}, 13^{25}, 14^{29}, 15^{27}$ \\
\hline 2 & $2,3,4,5,6^{3}, 7,8^{3}, 9^{3}$ & 6 & $2,3,4,5,6^{4}, 7^{2}, 8^{7}, 9^{9}, 10^{12}, 11^{16}, 12^{26}, 13^{26}, 14^{32}, 15^{32}, 16^{8}$ \\
\hline 3 & $2,3,4,5,6^{4}, 7^{2}, 8^{6}, 9^{8}, 10^{8}, 11^{10}, 12^{9}$ & 7 & $2,3,4,5,6^{4}, 7^{2}, 8^{7}, 9^{9}, 10^{12}, 11^{16}, 12^{26}, 13^{26}, 14^{33}, 15^{33}, 16^{11}$ \\
\hline 4 & $2,3,4,5,6^{4}, 7^{2}, 8^{7}, 9^{9}, 10^{11}, 11^{15}, 12^{22}, 13^{20}, 14^{16}, 15^{2}$ & 8 & $2,3,4,5,6^{4}, 7^{2}, 8^{7}, 9^{9}, 10^{12}, 11^{16}, 12^{26}, 13^{26}, 14^{33}, 15^{33}, 16^{12}$ \\
\hline
\end{tabular}

Table 3. The algebra obtained from the vacuum character for the coset model SU(3) $k / \mathrm{SU}(3)$ for various $k$ values. The algebra stabilizes by $k=8$.

\section{Generalized Casimir current algebra}

In this section, we write down the explicit form of the currents for the stringy coset in eq. (2.2). This coset and the associated current algebra have been studied in refs. [16, 17, 30, 31]. We review the known facts about the current algebra for this coset at arbitrary level $k$ in section 3.1. We are interested in the current algebra in the limit of large level $k$. We show in section 3.2 that in this limit, the coset theory reduces to a theory of free bosons. We also demonstrate that the number of currents grows with spin as expected from the vacuum character calculation in the previous section. We do this for the $N=2$ and $N=3$ cases in sections 3.2.1 and 3.2.2 respectively. Extrapolating from these results, we write down the general form of the current algebra generators in the large $N$ limit in section 3.2.3. We will identify the simplest of these generators with the free field realization of the $\mathcal{W}_{\infty}^{e}[1]$ algebra. The algebra $\mathcal{W}_{\infty}^{e}[\mu]$ is an infinite-dimensional sub-algebra of $\mathcal{W}_{\infty}[\mu]$, which consists of fields of even spin only [32-35]. In section 3.3, we will show that a subset of the generators of our stringy coset can be arranged in representations of $\mathcal{W}_{\infty}^{e}[\mu]$. This is evocative of the higher spin square which is the symmetry algebra of the large $N$ symmetric product orbifold theory [36]. We will remark on this correspondence between the generators of the higher spin square and the coset theory in section 3.3.

Here, we first establish background facts that we need to determine the currents for the coset $\mathrm{SU}(N)_{k} / \mathrm{SU}(N)$. For any coset algebra $G / H$, the generators are the currents of $G$ that commute with that of $H$. The generators of our coset algebra are composed from the $\mathrm{SU}(N)_{k}$ generators: $J^{a}$, where $a$ varies from 1 to $N^{2}-1$. These affine algebra generators, that transform in the adjoint representation of $\mathrm{SU}(N)$, satisfy the following operator product expansion:

$$
J^{a}(z) J^{b}(w)=\frac{-k \delta_{a b}}{(z-w)^{2}}+\frac{f_{a b c} J^{c}}{(z-w)}+\cdots .
$$

Repeated indices will always imply summation, regardless of the placement of the indices. The generators of the coset algebra are those operators of $\mathrm{SU}(N)_{k}$ that commute with the $\mathrm{SU}(N)$ currents, which are given by the zero modes of the affine currents. Thus, if $Q(z)$ is a generator of the coset algebra, then

$$
\left[J_{a}^{0}, Q(z)\right]=0 .
$$

As is shown in section 7.2.1 of ref. [16], this implies that $Q(z)$ must be a differential polynomial invariant in the $\mathrm{SU}(N)$ currents. The first such invariant is the stress-energy 
tensor

$$
T(z) \sim \frac{1}{2}: J^{a}(z) J^{b}(z):
$$

which is the quadratic Casimir of $\mathrm{SU}(N)$ defined up to an overall normalization and : $\cdots$ : symbol denotes normal ordering. It is a quasi-primary field of conformal dimension two. We will find that the coset algebra currents, in general, take a simple form in the quasiprimary basis. A quasi-primary field is defined as having the following commutator with the Virasoro modes of the stress-energy tensor

$$
\left[L_{m}, Q_{n}(z)\right]=\{n-(d-1) m\} Q_{n+m}
$$

where $m \in\{-1,0,1\}$. Here, $d$ is the conformal dimension of the field. A primary field on the other hand obeys eq. (3.4) for all mode numbers $m$.

As is well-known, the Casimir invariants are independent symmetric polynomial invariants of $\mathrm{SU}(N)$. In general, the number of polynomial differential invariants for a group, which is the set of possible currents for the coset CFT, is much larger.

\subsection{General $k$ and $k=1$}

At general level $k$ the stress energy tensor is given by

$$
T=\frac{-1}{2(k+N)}: J^{a} J^{a}:
$$

The coset currents for spin 3 and 4 for general level $k$ have been written down in ref. [17], which we now review. Any current can be formed using the generators $J_{a}$ and their derivatives. At any level $k$ and for any $N(>2)$ there is a spin 3 current of the form

$$
Q_{3}=\alpha d_{a b c}: J^{a} J^{b} J^{c}: .
$$

Here, $\alpha$ is a normalization factor that is given up to a constant by

$$
\alpha^{2}=\frac{N}{(k+N)^{2}(N+2 k)\left(N^{2}-4\right)},
$$

and $d_{a b c}$ is the third-order invariant symmetric tensor for $\mathrm{SU}(N)$. The above operator is, therefore, proportional to the third order Casimir of $\mathrm{SU}(N)$. The normal ordering for three fields is defined as

$$
: J^{a} J^{b} J^{c}:=: J^{a}: J^{b} J^{c}::
$$

and in a similar manner for operators consisting of more fields. Note that at spin 3 , this is the unique current that can be formed. The invariant : $J^{a} \partial J^{a}$ : is a total derivative of the stress-energy tensor : $J^{a} J^{a}$ : and, therefore, does not count as an independent current.

Two primary spin 4 currents were found for general $k$. The first field is

$$
\frac{1}{4(k+N)}\left\{: 2 \partial^{2} J^{a} J^{a}:-3: \partial J^{a} \partial J^{a}:\right\}+\beta: T T:+\gamma: \partial^{2} T:,
$$

where $\beta$ and $\gamma$ are numerical factors dependent on $N$ and $k$. The second field is given by

$$
Q_{4}=\alpha(k+N) d_{a b c d}: J^{a} J^{b} J^{c} J^{d}:,
$$


where $d_{a b c d}$ is the fourth-order invariant symmetric traceless tensor of $\mathrm{SU}(N)$. In general, for $\mathrm{SU}(N)$, there are $N-1$ primitive $d$-tensors of order $2, \cdots, N$. Each of these corresponds to a current. As we will show below, however, there are other $\mathrm{SU}(N)$ tensor invariants relevant to constructing coset currents.

The spin 4 field in eq. (3.10) occurs in the OPE of $Q_{3}(z)$ and $Q_{3}(w)$ :

$$
\begin{aligned}
Q_{3}(z) Q_{3}(w)= & \frac{c / 3}{(z-w)^{6}}+\frac{2 T(w)}{(z-w)^{4}}+\frac{\partial T(w)}{(z-w)^{3}} \\
& +\frac{1}{(z-w)^{2}}\left\{\frac{32}{22+5 c}: T(w) T(w):+\frac{3(c-2)}{10 c+44} \partial^{2} T(w)+Q_{4}(w)\right\} \\
& +\frac{1}{z-w}\left\{\frac{16}{22+5 c}: \partial(T(w) T(w)):+\frac{c-6}{15 c+66} \partial^{3} T(w)+\frac{1}{2} \partial Q_{4}(w)\right\} \\
& +\cdots
\end{aligned}
$$

A well-known result is that for the coset $\mathrm{SU}(N)_{1} / \mathrm{SU}(N)$, primary fields with spin higher than $N$ are either null or vanish and that there is only a single field at a given spin, so that the algebra becomes identical to the $W_{N}$ algebra. Let us show this for $N=3$, for the spin 4 operators. The field in eq. (3.10) vanishes for $\mathrm{SU}(3)$ as the tensor $d_{a b c d}$ collapses to zero for $N<4$. The second spin 4 field in eq. (3.9) can be written as

$$
Q_{4}^{N=3}=: T T:-\frac{33+31 k}{12(k+3)^{2}}: \partial J^{a} \partial J^{a}:-\frac{6+4 k}{9+3 k} \partial^{2} T .
$$

While in ref. [17] it was shown that the field in eq. (3.12) vanishes upon using an explicit realization of the Kac-Moody algebra in terms of free boson vertex operators (so that the Sugwara construction for $\mathcal{W}_{N}$ maps to the free field Miura realization), we can also directly compute the two-point function for $Q_{4}^{N=3}(z)$. This is given by

$$
\frac{k\left(-99-60 k+97 k^{2}+62 k^{3}\right)}{(3+k)^{4}}
$$

omitting overall numerical coefficients. The only non-zero integer value for which this vanishes and the primary field $Q_{4}^{N=3}(z)$ becomes null is $k=1$.

\subsection{The limit $k \rightarrow \infty$}

In this section, we will construct the coset currents in the large $k$ limit, which is the main objective of this paper. We will work at finite $N$ and then extrapolate our results to large $N$. The coset currents for finite $k$ explicitly depend on $k$. To remove this dependence, we will redefine the $\mathrm{SU}(N)_{k}$ generators as follows

$$
J^{a} \rightarrow \frac{1}{\sqrt{k}} J^{a}
$$

As a result of this redefinition the stress-energy tensor in eq. (3.5) becomes

$$
T=-\frac{1}{2} J^{a} J^{a}
$$


in terms of the new generators. Similarly the spin 3 current in eq. (3.6) (and other higher spin currents) become $k$-independent under this redefinition. The OPE in eq. (3.1) between the Kac-Moody currents becomes

$$
J^{a}(z) J^{b}(w)=\frac{-\delta_{a b}}{(z-w)^{2}},
$$

since the single-pole term is now suppressed by $\sqrt{k}$. The currents therefore, become essentially free in the large $k$ limit and the theory behaves like a theory of $N^{2}-1$ free bosons. To maintain continuity with finite $k$, we will continue to take the coset currents to be $\mathrm{SU}(N)$ invariants. Thus we retain the $\mathrm{SU}(N)$ as a global symmetry of the theory.

The behavior of the theory in the large $k$ limit can also be motivated as follows. The $\mathrm{SU}(N)_{k}$ current algebra can be written in terms of $N-1$ bosons and so-called "generating" parafermions [41]. The bosonic fields are denoted by $\phi_{i}$, where $1 \leq i \leq N-1$ and expressed as a vector $\phi$. The parafermions $\psi_{\alpha}$ are fractional spin fields associated with the root lattice of $\mathrm{SU}(N)$. Thus, here $\alpha$ labels the roots of $\mathrm{SU}(N)$. The conformal dimension of these fields is given by

$$
\Delta\left(\psi_{\alpha}\right)=1-\frac{\alpha^{2}}{2 k}=1-\frac{1}{k}
$$

where we have used the normalization $\alpha^{2}=2$. In terms of these parafermions $\psi_{\alpha}$ and the bosonic field $\phi$ the $\mathrm{SU}(N)_{k}$ generators take the form

$$
J_{i}(z) \sim \sqrt{k} \alpha_{i} \cdot \partial_{z} \phi
$$

when $J_{i}(z)$ belongs to the Cartan sub-algebra and the form

$$
J_{\alpha}(z) \sim \sqrt{k} \psi_{\alpha} \exp [i \alpha \cdot \phi(z) / \sqrt{k}]
$$

for the rest of the generators corresponding to the $N^{2}-N$ roots of $\mathrm{SU}(N)$. For level $k=1$, the parafermions $\psi_{\alpha}$ have vanishing dimension and the generators reduce to the usual vertex operator representation of the current algebra in terms of $N-1$ bosonic fields. As $k \rightarrow \infty$, the parafermions are promoted to bosons (of spin one). The exp term in eq. (3.19) reduces to one and the form of the generators $J_{\alpha}(z)$ become similar to $J_{i}(z)$. This is often referred to in the literature [13] as flattening of the $\mathrm{SU}(N)_{k}$ algebra in the large level limit to a $\mathrm{U}(1)^{N^{2}-1}$ algebra.

In the following, we will write down the generators of the coset algebra in the infinite level limit for the cases $N=2$ and $N=3$. We will denote a quasi-primary field of spin $s$ by $Q_{s}$ and a primary field by $P_{s}$. The associated $N$ value should be clear from context. We will always define the fields up to an overall normalization. To find the quasi-primary and primary currents, we used the Mathematica package OPEdefs [37].

\subsection{1 $\quad N=2$}

The $N=2$ case was studied in ref. [19]. A set of classical currents for the stringy $\mathrm{SU}(2)$ coset can be obtained by acting on the Casimir invariant $\operatorname{Tr}(J J)$ by derivatives. These currents are of the form

$$
\operatorname{Tr}\left(\partial^{\mu} J \partial^{\nu} J\right)
$$


In addition to the Casimir invariant, $\mathrm{SU}(2)$ has cubic invariants given by

$$
\operatorname{Tr}\left(\left[\partial^{\mu} J, \partial^{\nu} J\right] \partial^{\gamma} J\right) \quad \forall \mu \neq \nu \neq \gamma .
$$

We can count the number of these invariants. The number of bilinear terms is the number of ways one can divide an integer into exactly two parts. The number of trilinear terms is given by the generating series for the number of ways to divide an integer into three distinct parts. The number of ways to divide an integer into $p$ distinct parts is given by the generating function:

$$
\frac{q^{p(p+1) / 2}}{(1-q)\left(1-q^{2}\right) \cdots\left(1-q^{p}\right)} .
$$

To get the independent terms we remove the total derivatives. Then the generating function for the classical currents is given by

$$
\frac{q^{2}}{\left(1-q^{2}\right)}+\frac{q^{6}}{\left(1-q^{2}\right)\left(1-q^{3}\right)}=q^{2}+q^{4}+2 q^{6}+2 q^{8}+q^{9}+2 q^{10}+q^{11}+2 q^{12}+\cdots .
$$

To find the quantum algebra of the coset, we have to find the primary completion of the classical currents. Relations between the invariant tensors of $\mathrm{SU}(2)$ will make some of these currents vanish. This together with the presence of null states will truncate the set of infinite currents to the finite set listed in eq. (2.15), derived from the vacuum character. In fact, it can be shown that the first vanishing state arises at the order at which a syzygy (a relation between the invariants) is present. For more details regarding this, the reader is referred to ref. [19].

We now write down the explicit form of the currents up to an overall normalization. Our strategy for determining the explicit form of the currents is as follows. We first write down the most general form of a current of given spin $s$ and of a given order $p$ in the $J^{a}$. For spin $s=6$, for example, the most general form of a current of order 2 is

$$
\alpha: \partial^{3} J^{a} \partial J^{a}:-\beta: \partial^{2} J^{a} \partial^{2} J^{a}:-\gamma: \partial^{4} J^{a} J^{a}:,
$$

while the most general form of a current of order 3 is

$$
\alpha \epsilon_{a b c}: J^{a} \partial J^{b} \partial^{2} J^{c}:
$$

Here, the coefficients $\alpha, \beta, \gamma$ are undetermined. We then fix these coefficients by demanding that these currents be quasi-primary: that is, the OPE of the stress-energy tensor with the current have a vanishing third-order pole. Note that this procedure can result in multiple soultions for the coefficients which simply means that there are distinct quasi-primaries.

Once we have determined a quasi-primary field, we can find the associated primary field. We add all possible terms of given spin (with undetermined coefficients) and of arbitrary order in the $J^{a}$ to the quasi-primary field and demand that the OPE with the stress-energy tensor not have any poles of order greater than 2 . This procedure will give a solution for the coefficients only when such a primary field exists, although it can be null. Our aim in this subsection is to show that the number of primary fields determined by 
this method matches the one in table 2 for $N=2$. In principle, once the primary field is determined we can check if it is null by computing its two-point function with itself; the two-point function can be read off from OPE of field with itself. However, this procedure is computationally intensive for fields with large spin. Hence, it is impractical to use it to determine the primary field with the largest spin. We stress, however, that because of the finite central charge of the coset CFT at finite $N$, we expect that there a finite number of non-null primary fields that can be constructed from the (infinite number of) $\mathrm{SU}(N)$ invariants.

The stress energy tensor of the coset is given by eq. (3.15). There is no spin 3 current. We have a single primary field of dimension 4 which is of the form

$$
P_{4}=\left(\frac{1}{2}: \partial^{2} J^{a} J^{a}:-\frac{3}{4}: \partial J^{a} \partial J^{a}:\right)-\frac{9}{37}: T T:+\frac{30}{37} \partial^{2} T .
$$

Note that the first term in brackets is a quasi-primary field of dimension 4 while the rest are correction terms to make the field primary. Next we have two primary fields of spin 6 . The first is of the form in eq. (3.20) and is given by

$$
\begin{aligned}
P_{6,1}= & : \partial^{3} J^{a} \partial J^{a}:-: \partial^{2} J^{a} \partial^{2} J^{a}:-\frac{1}{10}: \partial^{4} J^{a} J^{a}: \\
& +\alpha: T T T:+\beta: \partial^{2} T T:+\gamma: \partial T \partial T:+\delta: Q_{4} T:+\epsilon \partial^{2} Q_{4}+\zeta \partial^{4} T
\end{aligned}
$$

The term in the first line of the r.h.s. is the associated quasi-primary field. The coefficients $\alpha, \beta, \cdots$ are given in appendix $\mathrm{B}$. The second field is of the form eq. (3.21) and is given by

$$
P_{6,2}=\epsilon_{a b c}: J^{a} \partial J^{b} \partial^{2} J^{c}:
$$

where $\epsilon_{a b c}$ is the Levi-Civita tensor. The expression as written above is already a primary field of dimension 6 and does not need any correction terms. Continuing in this manner, one can write down all operators of the algebra. As stated above, the primary fields start becoming null from spin 11 and the algebra thus consists of a finite number of fields.

\subsection{2 $N=3$}

For the stringy $\mathrm{SU}(3)$ coset, the classical currents related to the Casimir invariants are of the form

$$
\begin{aligned}
\operatorname{Tr}\left(\partial^{\mu} J \partial^{\nu} J\right) & \equiv \partial^{\mu} J^{a} \partial^{\nu} J^{a}, \\
\operatorname{Tr}\left(\left\{\partial^{\mu} J, \partial^{\nu} J\right\} \partial^{\gamma} J\right) & \equiv d_{a b c} \partial^{\mu} J^{a} \partial^{\nu} J^{b} \partial^{\gamma} J^{c} .
\end{aligned}
$$

Below we list all the currents up to spin 6 . The lowest spin operators having the structure in eq. (3.29) are the stress-energy tensor and the spin 3 Casimir current:

$$
P_{3}=d_{a b c}: J^{a} J^{b} J^{c}:
$$

The lowest spin currents with additional derivatives are the spin 4 current of the form

$$
Q_{4}=\frac{1}{2}: \partial^{2} J^{a} J^{a}:-\frac{3}{4}: \partial J^{a} \partial J^{a}:
$$


and the spin 5 current of the form

$$
Q_{5}=d_{a b c}\left(: \partial^{2} J^{a} J^{b} J^{c}:-\frac{3}{2}: \partial J^{a} \partial J^{b} J^{c}:\right) .
$$

At the next level there are two additional spin 6 currents of the form

$$
Q_{6,1}=: \partial^{3} J^{a} \partial J^{a}:-: \partial^{2} J^{a} \partial^{2} J^{a}:-\frac{1}{10}: \partial^{4} J^{a} J^{a}:
$$

and

$$
Q_{6,2}=d_{a b c}\left(: \partial^{3} J^{a} J^{b} J^{c}:-6: \partial^{2} J^{a} \partial J^{b} J^{c}:+6: \partial J^{a} \partial J^{b} \partial J^{c}:\right) .
$$

The currents as written are all quasi-primary, save for the spin 3 current $P_{3}$ which is primary. The primary completion of these fields appear in appendix B.

Apart from the Casimir invariants, there are other invariants as well for $\mathrm{SU}(3)$. The tensor $f_{a b c}$ is a skewsymmetric invariant and it leads to the currents of the form

$$
f_{a b c} \partial^{\mu} J^{a} \partial^{\nu} J^{b} \partial^{\gamma} J^{c} \forall \mu \neq \nu \neq \gamma
$$

Indeed the first such current is

$$
P_{6,3}=f_{a b c}: \partial^{2} J^{a} \partial J^{b} J^{c}:
$$

which is a primary field of dimension 6 . This accounts for three of the four spin 6 currents predicted by the vacuum character. Thus, by sprinkling derivatives on the symmetric and anti-symmetric tensor invariants of $\mathrm{SU}(3)$, we do not get all the expected currents.

To write the final spin 6 current it is useful to look at the primary fields in the theory. Primary fields of the coset CFT can be divided into two categories: those that are SU(3) singlets and those that are not. The vacuum character contains information about primary fields that are also SU(3) singlets. Taking the vacuum character in eq. (2.20) and expanding in Virasoro characters gives

$$
(1-q) V_{0}(q)+V_{2}(q)+V_{3}(q)+V_{4}(q)+V_{5}(q)+5 V_{6}(q)+3 V_{7}(q)+11 V_{8}(q)+\cdots .
$$

where $V_{h}(q)$ is the character of the Virasoro algebra Verma module

$$
V_{h}(q)=q^{h} \prod_{j=1}^{\infty} \frac{1}{1-q^{j}} .
$$

and we have assumed that the Virasoro characters are irreducible. From eq. (3.37), we can see that there should be a total of five primary fields of spin 6 , that are also $\mathrm{SU}(3)$ singlets. These include the fields that are composite operators.

Composite operators can be divided into two categories: those that are composed of $\mathrm{SU}(3)$ singlets and those that are composed of fields transforming non-trivially under $\mathrm{SU}(3)$. An example of the first kind of operator is the following composite spin 6 operator:

$$
P_{6,4}=: P_{3} P_{3}:+\alpha: T T T:+\beta: Q_{4} T:+\gamma: \partial^{2} T T:+\delta: \partial T \partial T:+\epsilon \partial^{2} Q_{4}+\zeta \partial^{4} T .
$$


This is a primary field if the coefficients $\alpha, \beta, \gamma, \delta, \epsilon, \zeta$ take the values

$$
\alpha=\frac{503}{372}, \beta=-\frac{33}{40}, \gamma=\frac{2627}{1240}, \delta=\frac{413}{124}, \epsilon=\frac{37}{80}, \zeta=-\frac{101}{310} .
$$

To find the other $\mathrm{SU}(3)$ invariants we look for primary fields that are not $\mathrm{SU}(3)$ singlets. A spin 2 field of this nature was introduced in ref. [17], and takes the form

$$
P_{2}^{a}=d_{b c}^{a}: J^{b} J^{c}:
$$

This is not the only possible primary field transforming in the $\mathrm{SU}(3)$ adjoint rep. Fields of the schematic form $d_{a b c}: \partial^{\mu} J^{a} \partial^{\nu} J^{b}$ : can be potential primaries. The operator

$$
P_{4}^{a}=d_{b c}^{a}\left(: \partial^{2} J^{b} J^{c}:-\frac{3}{2}: \partial J^{b} \partial J^{c}:\right)+\frac{3}{14}: \partial^{2} P_{2}^{a}:-\frac{5}{7}: P_{2}^{a} T: .
$$

is a (non-null) spin 4 primary. In fact, we can generate primaries from the skew-symmetric tensor invariant $f_{a b c}$ in the same manner. The field

$$
P_{3}^{a}=f_{b c}^{a}: \partial J^{b} J^{c}:
$$

is a primary operator of dimension three.

We can construct new $\mathrm{SU}(3)$ singlets from such primary fields. It will not, however, always be the case that the operators generated in this way are distinct to the singlets already constructed or are not null. The composite spin 4 primary

$$
: P_{2}^{a} P_{2}^{a}:-\frac{40}{31} \partial^{2} T-\frac{220}{93}: T T:
$$

is the same as the primary completion of the spin 4 field in eq. (3.31). However, the composite field

$$
P_{6,5}=: P_{2}^{a} P_{4}^{a}:+\frac{15}{7}: T T T:-\frac{17}{42}: \partial^{2} T T:+\frac{5}{21}: \partial T \partial T:+\frac{31}{14}: Q_{4} T:+\frac{62}{63} \partial^{4} T-\frac{527}{252} \partial^{2} Q_{4}
$$

is a new spin 6 primary field. It can be easily verified, using Mathematica, that the operators $P_{6,1}, P_{6,2}, P_{6,3}, P_{6,4}$ and $P_{6,5}$ are linearly independent. Note that we can construct another spin 6 singlet of the form : $P_{3}^{a} P_{3}^{a}$ :, but this operator is a linear combination of $P_{6,1}, P_{6,2}$ and $P_{6,5}$.

The primary field in eq. (3.45) is a composite field as far as the CFT is concerned, but is an independent $\mathrm{SU}(N)$ invariant. It will, therefore, be counted by the vacuum character of the coset CFT. The spin 6 fields that contribute to the vacuum character are thus: $P_{6,1}, P_{6,2}, P_{6,3}$ and $P_{6,5}$. Note that the field $P_{6,4}$ is a "double-trace" $\mathrm{SU}(N)$ invariant and its contribution to the vacuum character has already been accounted for by $P_{3}$. The number of independent currents at spin 6 is thus four as predicted by the vacuum character. We see that unlike for the $N=2$ case, the $N=3$ stringy coset also needs composite currents to generate the full set of currents. Of course, the currents $T, P_{4}$ etc are also composites of the primary field $J^{a}$, but we use the word composite here to mean operators that are composites of primary fields that are themselves composite in $J^{a}$. It is not difficult to 
estimate the number of such composite currents, although it is hard to discern which of them are non-redundant $[43,44]$. For SU(3), invariant tensors that can be formed out of the composite operators, denoted here by $A^{a}, B^{a}, C^{a}$, take the form

$$
d_{a b} A^{a} B^{b}, \quad d_{a b c} A^{a} B^{b} C^{c}
$$

The growth rate for such composite operators (with increasing spin) exceeds the growth rate for generators predicted by the vacuum character in eq. (2.20). However, it has to be checked on a case-by-case basis which of these fields are independent and contribute to the vacuum character.

All the generators that we have constructed for infinite $k$ are also present at finite $k$ (for $k \geq 3$ ). It is worthwhile to write down the exact form of some of these generators for finite $k$. At finite $k$, the spin 4 primary field takes the form

$$
P_{4}(k)=Q_{4}-\frac{9(3+k)^{2}}{33+31 k} \partial T+\frac{30(3+k)^{2}}{33+31 k}: T T: .
$$

The bilinear spin 6 primary current takes the form

$$
\begin{aligned}
P_{6,1}(k)= & Q_{6,1}-\frac{21 \alpha^{2}\left(-129+106 k+55 k^{2}\right)}{5 \beta \gamma \delta}: \partial^{2} T T:-\frac{42 \alpha^{2}(1+k)(6+k)}{\beta \gamma \delta}: \partial T \partial T: \\
& +\frac{21 \alpha}{5 \beta}: Q_{4} T:+\frac{2 \alpha^{2}\left(-219+1126 k+790 k^{2}\right)}{5 \beta \gamma \delta} \partial^{4} T-\frac{49 \alpha}{10 \beta} \partial^{2} Q_{4} \\
& -\frac{42 \alpha^{3}(3-7 k)}{\beta \gamma \delta}: T T T:
\end{aligned}
$$

where $\alpha=3+k, \beta=9+4 k, \gamma=1+5 k, \delta=51+31 k$. In the above equations, the stress-energy tensor is defined as in eq. (3.5) and the the currents $J^{a}$ are rescaled as $\sqrt{k} J^{a}$. The form of the quasi-primary operators is thus independent of $k$ for the bilinear currents. This is not true for the generator $P_{6,3}$. This current is not primary at finite $k$ and has to be modified in the following way to stay primary

$$
\begin{aligned}
P_{6,3}(k)= & f_{a b c}: \partial^{2} J^{a} \partial J^{b} J^{c}:-\frac{21 \alpha^{2}\left(1353+6518 k+2225 k^{2}\right)}{5 \beta \gamma \delta}: \partial^{2} T T: \\
& -\frac{42 \alpha^{2}\left(159+160 k+61 k^{2}\right)}{5 \beta \gamma \delta}: \partial T \partial T:+\frac{23 \alpha}{5 \beta}: Q_{4} T: \\
& +\frac{2 \alpha\left(-2529+8379 k+9668 k^{2}+2150 k^{3}\right)}{5 \beta \gamma \delta} \partial^{4} T+\frac{474+157 k}{30 \beta} \partial^{2} Q_{4} \\
& -\frac{2 \alpha^{3}(3+193 k)}{\beta \gamma \delta}: T T T: .
\end{aligned}
$$

Note that as $k \rightarrow \infty$ the fields $P_{4}(k)$ and $P_{6,1}(k)$ reduce to their corresponding counterparts in eq. (B.2) and eq. (B.4), while $P_{6,3}(k)$ becomes identical to eq. (3.36).

\subsubsection{Large $N$}

At large $N$, the currents that follow from the symmetric $d$-tensor invariants are given by

$$
d_{a b c \cdots} \partial^{\mu} J^{a} \partial^{\nu} J^{b} \partial^{\gamma} J^{c} \cdots
$$




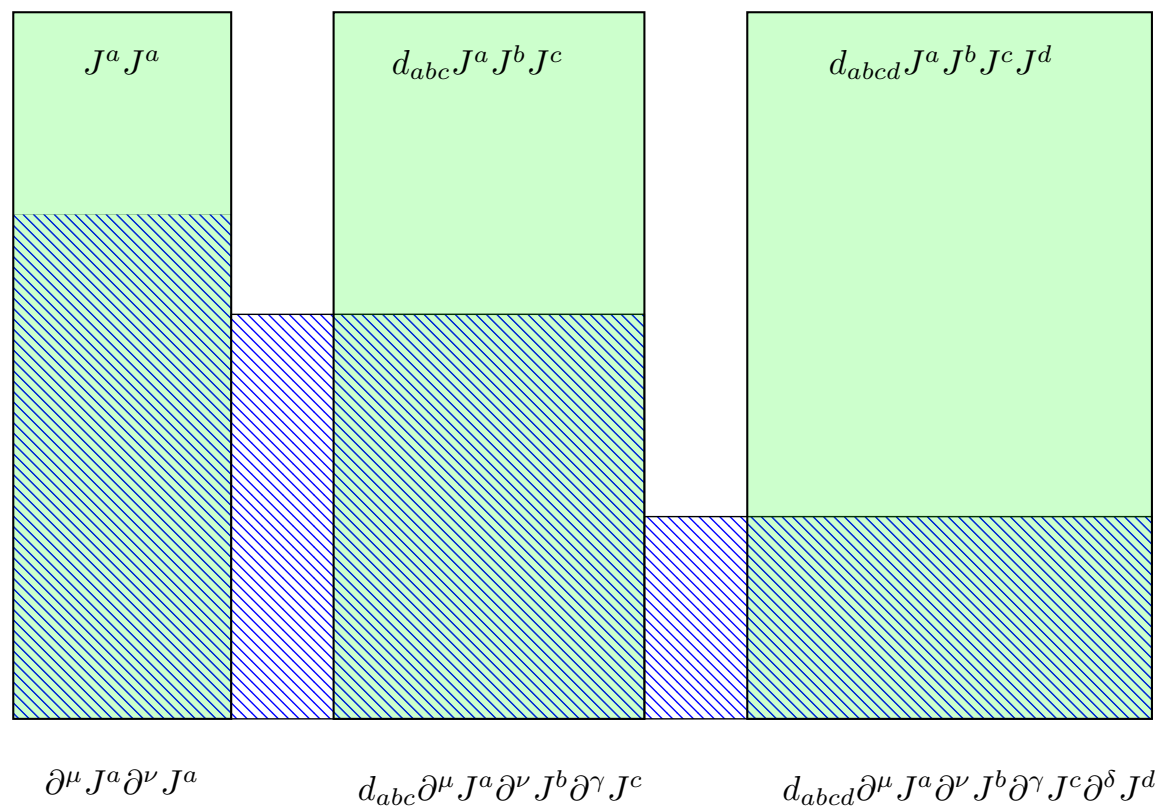

Figure 1. The operators in the top-most row correspond to the $\mathrm{SU}(N)$ Casimir invariants. A subset of generators for the stringy coset algebra is generated by acting by derivatives on the constituent terms of each Casimir operator. The cross-hatched area denotes the operators that are null and not part of the algebra for a given level $k$ and $N$. For $k=1$, only the top row of operators is not null and corresponds to the $W_{N}$ algebra. Increasing the level $k$ reduces the cross-hatched area but does not eliminate it completely even as $k$ tends to $\infty$. Increasing $N$ corresponds to adding more columns and also reducing the cross-hatched area. As we take $N$ to $\infty$, with $k$ already taken to $\infty$, null states disappear from the first column and the algebra becomes $\mathcal{W}_{\infty}^{e}[1]$

For $\mathrm{SU}(N)$ there is a single Casimir invariant at orders $2, \cdots, N$. Since, the tensors $d_{a b c \cdots}$ are totally symmetric, the generating function for an order $p$ current of the form in eq. (3.50) is given by the number of ways to divide an integer into $p$ parts. At infinite $N$, there are no relations between the $\mathrm{SU}(N)$ invariant tensors. Hence, it is reasonable to assume that at large $N$, there are no null fields in this set of currents. Then at infinite $N$, the generating function for the generalized Casimir currents is

$$
\sum_{p=2}^{\infty} \frac{q^{p}}{\prod_{k=2}^{p}\left(1-q^{k}\right)}=\prod_{n=2}^{\infty} \frac{1}{\left(1-q^{n}\right)}-1 .
$$

Thus, the number of independent currents grows at least as fast as $\exp (\sqrt{n})$. In summing up the l.h.s., we have used the Cauchy identity [38]

$$
1+\sum_{p=1}^{\infty} \frac{q^{p}}{\prod_{k=1}^{p}\left(1-q^{k}\right)}=\prod_{n=1}^{\infty} \frac{1}{\left(1-q^{n}\right)} .
$$

The identity in eq. (3.51) then follows by multiplying both sides by $(1-q)$ and rearranging.

Clearly as we saw from the above examples of SU(2) and SU(3), these are not the only possible currents. For the group $\mathrm{SU}(N)$, there are skew-symmetric invariant tensors $f_{a b c \ldots}$ 
of order $3,5, \cdots, 2 N-1$ which lead to antisymmetric currents. In the literature, these skew-symmetric tensors are also known as $\Omega$ tensors [39, 40]. The total number of such states as $N \rightarrow \infty$, assuming no null states is given by

$$
\sum_{\substack{k=3 \\ k \in \text { odd }}}^{\infty} \frac{q^{k(k+1) / 2}}{\left(1-q^{2}\right) \cdots\left(1-q^{k}\right)}=\frac{1-q}{2}\left\{\prod_{n=1}^{\infty}\left(1+q^{n}\right)-\prod_{n=1}^{\infty}\left(1-q^{n}\right)\right\}-q .
$$

The equality of the two sides follows from a second identity of Cauchy's:

$$
\sum_{k=0}^{\infty} \frac{q^{k(k-1) / 2} x^{k}}{(1-q) \cdots\left(1-q^{k}\right)}=\prod_{n=0}^{\infty}\left(1+q^{n} x\right)
$$

Setting $x=q$ first and then $x=-q$ will give two sets of equalities. Subtracting the second from the first, multiplying both sides by $(1-q)$ and rearranging gives the equality in eq. (3.53).

As we saw for finite $N$, composite currents are also present for the coset CFT. In general, it is hard to count these currents. Invariants composed of primary fields that transform non-trivially under $\mathrm{SU}(N)$ do not always lead to new or non-null currents. This happens because of the identities that exist between $\mathrm{SU}(N)$ tensors (see, for example, appendix B of [17]) at finite $N$.

\subsection{Relation with $\mathcal{W}_{\infty}^{e}[1]$ and the higher spin square}

We now focus on the currents bilinear in the $J^{i}$ 's. As we saw for finite $N$, the independent bilinear currents at any value of the level $k$ have even spin only with multiplicity one at each spin. In the quasi-primary basis, these currents do not change with increasing $k$ or $N$, since only the overall normalization changes. (In the primary basis, this is no longer true). In the large level limit and at finite $N$, the coset theory reduces to that of $N^{2}-1$ free bosons, hence the bilinear currents can be identified with a realization of the $\mathcal{W}_{\infty}^{e}[1]$ algebra at central charge $N^{2}-1$. As is well known, such a realization is a finite truncation of the $\mathcal{W}_{\infty}^{e}[1]$ algebra $[45,46]$. As we take $N \rightarrow \infty$, we recover the full $\mathcal{W}_{\infty}^{e}[1]$ algebra.

It is natural to ask whether the higher-order generators of the stringy coset algebra as arranged in figure (1) can be identified with representations of the $\mathcal{W}_{\infty}^{e}[1]$ algebra. The OPE of any $\mathcal{W}_{\infty}^{e}[1]$ operator, which are the bilinear operators, with a generator of order $p$ gives rise to operators of the same order. For example, the OPE of any bilinear term of the form : $\partial^{\mu} J^{a} \partial^{\nu} J^{a}$ : with a generic trilinear term is

$$
\begin{aligned}
& : \partial^{\mu} J^{a} \partial^{\nu} J^{a}(z):: d_{b c d} \partial^{\alpha} J^{b} \partial^{\beta} J^{c} \partial^{\gamma} J^{d}(w): \\
& \quad \sim \frac{\delta^{a b} \delta^{a c} d_{b c d} \partial^{\gamma} J^{d}(w)}{(z-w)^{\mu+\nu+\alpha+\beta+4}}+\frac{\delta_{b}^{a} d_{b c d}: \partial^{\nu} J^{a}(z) \partial^{\beta} J^{c}(w) \partial^{\gamma} J^{d}(w):}{(z-w)^{\mu+\alpha+2}} \\
& \quad \sim \frac{d_{b c d}: \partial^{\nu} J^{b}(w) \partial^{\beta} J^{c}(w) \partial^{\gamma} J^{d}(w):}{(z-w)^{\mu+\alpha+2}}+\frac{d_{b c d}: \partial^{\nu+1} J^{b}(w) \partial^{\beta} J^{c}(w) \partial^{\gamma} J^{d}(w):}{(z-w)^{\mu+\alpha+1}}+\cdots
\end{aligned}
$$

We have written the OPE schematically omitting numerical coefficients in all terms and writing only a single representative term for different possible ways of contracting. The 
first term in the second line vanishes because the tensor $d_{b c d}$ is traceless. Thus we are only left with trilinear operators in the OPE. The same logic applies to the OPE of any $p$-th order operator with a bilinear operator. The operators in each column of figure (1) thus fall into a representation of $\mathcal{W}_{\infty}^{e}[1]$.

Let us identify the $\mathcal{W}_{\infty}^{e}[1]$ representation that corresponds to each column of operators in figure (1). We use the standard coset notation for representations of the $\mathcal{W}_{\infty}^{e}[1]$ alegbra. Conventional $\mathcal{W}$-algebras that we deal with in this paper are the symmetry algebras of cosets of the form $\frac{\mathfrak{g}_{k} \otimes \mathfrak{g}_{1}}{\mathfrak{g}_{k+1}}$. The notation $\left(\Lambda_{+} ; \Lambda_{-}\right)$is used to denote a representation of a $\mathcal{W}$-algebra where $\Lambda_{+}$is a representation of $\mathfrak{g}_{k}$ and $\Lambda_{-}$is a representation of $\mathfrak{g}_{k+1}$. Then, a order $p$ column corresponds to the representation $\left(\left[0^{p-1}, 1,0, \ldots, 0\right] ; 0\right)$ of $\mathcal{W}_{\infty}^{e}[1]$. The wedge character of a representation $R$ of $\mathcal{W}_{\infty}^{e}[\mu]$ is given by

$$
q^{\frac{\mu}{2} B\left(R^{T}\right)} \chi_{R^{T}}^{U(\infty)}
$$

where $R^{T}$ is the transpose of the representation $R, B\left(R^{T}\right)$ is the number of boxes in the Young tableaux of $R^{T}$ and $\chi^{U(\infty)}$ is the associated Schur function (See [36, 47] for details). Thus the wedge character of the representation $\left(\left[0^{p-1}, 1,0, \ldots, 0\right] ; 0\right)$ is given by

$$
b_{\left(\left[0^{p-1}, 1,0, \ldots, 0\right] ; 0\right)}^{(\text {wedge }[\lambda=1]}=\frac{q^{p / 2} q^{p / 2}}{\prod_{k=1}^{p}\left(1-q^{k}\right)}
$$

which is the same as the generating function of a column of operators with order $p$. Note that these are the same representations that constitute the higher spin square, the algebra of the large $N$ symmetric product orbifold.

The operators of the coset algebra look very different to corresponding operators of the higher spin square algebra. Nevertheless, the number of operators (and hence the character of the representation) is the same in a column of the coset algebra whose highest weight state is an operator of the form $d_{a b \ldots} J^{a} J^{b} \ldots$ of order $p$ and in a column of the higher spin square (See figure 2) whose highest weight state is of the form $J^{a} J^{a} \ldots$ of the same order $p$. Identical characters imply identical representations, since a representation of an algebra has a unique character. Thus, it is possible that the subset of generators of the coset algebra that are present in figure (1) is isomorphic to the higher spin square.

Generators of the higher spin square can also be organized in terms of a $\mathcal{W}_{\infty}[0]$ algebra which is called the horizontal sub-algebra in ref. [36]. In the coset case, this means that there should exist a change of basis for the $\mathrm{SU}(N)$ currents, such that in the new basis the generators in the top row of figure (1) close at infinite $k$. It is possible that such a basis exists, since the coset theory also has a free fermion formulation at $c=N^{2}-1$. As is well known, the $\mathcal{W}_{\infty}[0]$ algebra can be expressed in terms of free fermions [48].

A further check of whether the higher spin square is embedded in the coset algebra, is to see whether the set of operators in figure (1) have closed commutation relations. At finite $N$, this is not true and the OPE of any two such operators will in general contain terms containing composite operators of the kind defined in eq. (3.46). Thus at finite $N$, the algebra does not close on the set of symmetric operators alone. However, terms that occur in the OPE are scaled by different powers of $N$. It is, thus, possible that some of 
these terms are suppressed in the large $N$ limit. It will require a careful analysis of the OPEs to see whether the set of symmetric operators is indeed isomorphic to the higher spin square.

Next we look at operators of the generic form : $f_{b c d} \ldots \partial^{\alpha} J^{b} \partial^{\beta} J^{c} \partial^{\gamma} J^{d}(w) \cdots$. The generating function for this set of operators is given by eq. (3.22). Interpreting this as a wedge character of $\mathcal{W}_{\infty}^{e}[1]$, we find

$$
\frac{q^{p(p+1) / 2}}{\prod_{k=1}^{p}\left(1-q^{k}\right)}=\frac{q^{p / 2} q^{p^{2} / 2}}{\prod_{k=1}^{p}\left(1-q^{k}\right)}=b_{([p, 0, \ldots, 0] ; 0)}^{(\text {wedge }[\lambda=1]},
$$

that it corresponds to the representation $([p, 0, \ldots, 0] ; 0)$. All the "elementary operators" of the stringy coset algebra can thus be organized into representations of $\mathcal{W}_{\infty}^{e}[1]$.

\section{Discussion}

In this paper, we have examined the coset in eq. (1.2) in the free field limit, which is equivalent to its zero coupling limit. On the basis that the central charge of this coset scales as $N^{2}$, it has generally been expected that this coset is dual to a string theory in the bulk. We computed the vacuum character for the coset at finite $N$ and found that the currents of the symmetry algebra indeed exhibit an exponential growth with the spin. The $\mathcal{W}$-algebra of the coset in eq. (1.2) in the free field limit is the same as the $\mathcal{W}$-algebra of the coset in eq. (2.2) in the large $k$ limit. We have written down the explicit form of the low-dimension currents for the second coset model. As we saw in the text, the currents of this coset are simply $\mathrm{SU}(N)$ invariants composed out of differential operators of the form $\partial^{\mu} J^{a}$, where the $J^{a}$ are $\mathrm{SU}(N)_{k}$ currents. As such, this coset theory is an exact analog in two dimensions of $\mathrm{SU}(N)$ Yang-Mills theories in higher dimensions, with the addition of Virasoro symmetry.

The zero coupling limit of supersymmetric Yang-Mills theory in four dimensions is expected to be dual to tensionless string theory on the $A d S_{5}$ background $[49,50]$. It is, therefore, of interest to ask what the bulk dual of our coset model in the free field limit is. We know, from considerations of the D1-D5 system in Type IIB string theory, that it is a symmetric product orbifold theory that is expected to be dual to string theory on the $A d S_{3}$ background. Indeed, there has been much recent work in this direction clarifying aspects of this duality between tensionless strings and the free symmetric product orbifold theory [51-54]. It is not clear where the coset theories, we have considered in this paper, fit into this picture. Though on general grounds we expect the large $N$ coset theory to have a $A d S_{3}$ string dual, further analysis would be needed to pinpoint such a dual. A more detailed understanding of the moduli space of $A d S_{3}$ string theories would be useful [55]. Further hints may come from integrability $[56,57]$.

How does the more general $\mathcal{W}$-algebra of the stringy coset models relate to the $\mathcal{W}_{\infty}[\mu]$ symmetry of the vector coset models? We found that in the free field limit, the algebra $\mathcal{W}_{\infty}^{e}[1]$ is a sub-algebra of the full coset algebra. Further, there is a distinguished set of generators of the coset algebra that can be arranged into representations of $\mathcal{W}_{\infty}^{e}[1]$. Operators that are directly derived from the symmetric tensor invariants of $\mathrm{SU}(N)$ can be 
arranged in the $\left(\left[0^{p-1}, 1,0, \ldots, 0\right] ; 0\right)$ representations of $\mathcal{W}_{\infty}^{e}[1]$. Operators that are related to antisymmetric invariants of $\mathrm{SU}(N)$ can be arranged in the $([p, 0, \ldots, 0] ; 0)$ representations of $\mathcal{W}_{\infty}^{e}[1]$. Since the first set of operators can be organized in the same set of representations of $\mathcal{W}_{\infty}^{e}[1]$ as the operators of the higher spin square, the symmetry algebra of the symmetric product orbifold CFT, we propose that this set of operators is identical to the higher spin square. The higher spin square also has a $\mathcal{W}_{\infty}[0]$ horizontal algebra, in addition to the vertical $\mathcal{W}_{\infty}^{e}[1]$ algebra. We have not explicitly identified this horizontal algebra in the coset case. In addition, we have not shown that the algebra of the set of symmetric operators closes by itself. It is important to do so, in order to confirm the identification of the coset sub-algebra with the higher spin square.

More broadly, one can ask why the higher spin square, whose generators are invariants of $S_{N}$, and the stringy coset algebra, whose generators are invariants of $\mathrm{SU}(N)$, should be related (in the large $N$ limit) to each other? In both cases, we have a $\mathcal{W}_{\infty}^{e}[1]$ subalgebra, and we are using free fields to construct (symmetric) representations of this algebra. It is not very hard to construct representations of $\mathcal{W}_{\infty}^{e}[1]$ : operators of the form $d_{a b c \ldots} \partial^{\mu} J^{a} \partial^{\nu} J^{b} \partial^{\gamma} J^{c} \ldots$ suffice with the one necessary condition that the tensor $d_{a b c \ldots}$ be totally symmetric and traceless. This condition leads to both the $S_{N}$ and $\mathrm{SU}(N)$ solutions, with the indices $a, b, c, \cdots$ taking values from 1 to $N$ for the $S_{N}$ case. This still leaves open the question of whether operators in the two sets of representations constitute identical algebras.

In addition to the "elementary" generators, the coset theory also has a large number of composite operators at general values of $N$. In this paper, we have not attempted to classify them in representations of $\mathcal{W}_{\infty}^{e}[1]$. It is obviously of interest to understand the nature of these generators to comprehend the full symmetry algebra of the stringy coset theory. The coset in eq. (1.2) is T-dual to a coset which is holographically dual to Vasiliev theory with a matrix extension. It would be interesting to explore the exact relation between this stringy coset and the matrix cosets [58]. In this paper, we have worked in the limit of zero coupling. However, in general the coset algebra depends on the parameter $\lambda$. It would be interesting to find how the algebra changes once the coupling is switched on. At certain values of non-zero $\lambda$, for example at $\lambda=1$, the coset theory has a formulation in terms of free bosons/fermions. This is also the point, where the symmetry algebra of the coset enhances to an $\mathcal{N}=1$ supersymmetric algebra. It would be nice to do an analysis similar to this paper for the $\lambda=1$ theory. More generally, Wolf space coset generalizations of eq. (1.2) can be studied in a similar manner.

\section{Acknowledgments}

We thank Rajesh Gopakumar, Yang-Hui He, Bogdan Stefanski and Alessandro Torrielli for discussions. We thank Matthias Gaberdiel for valuable comments on the first version of this paper. We thank Biswajit Ransingh for running a computer program for us at HRI, Allahabad. 


\section{A The $\mathrm{SU}(N)$ tensor invariants}

The tensors $d_{a b c d . .}$ are totally symmetric tensors which we have also chosen to be traceless. Suitable traceless symmetric tensors are defined in [39], where they are referred to as $t$-tensors.

We use the notation $\mathfrak{d}$ for the standard symmetric invariant tensors of $\mathrm{SU}(N)$. They are defined recursively [59] starting from the standard third-order symmetric tensor $\mathfrak{d}_{i j k}$. One can construct the tensor

$$
\mathfrak{d}^{(r+1)}{ }_{i_{1} \ldots i_{r+1}}=\mathfrak{d}^{(r)}{ }_{i_{1} \ldots i_{r-1} j} \mathfrak{d}^{(3)}{ }_{j i_{r} i_{r+1}} \quad, \quad r=3,4, \ldots .
$$

For $r \geq 3$, the above construction does not define totally symmetric tensors. The $\mathfrak{d}$-family of symmetric tensors is obtained by symmetrising over all free indices in (A.1).

The $\mathrm{SU}(n) d$ tensors are related to members of the $\mathfrak{d}$-family in the following way

$$
\begin{aligned}
d_{i j} & \sim \delta_{i j}, \\
d_{i j k} & \sim \mathfrak{d}_{i j k}, \\
d_{i j k l} & \sim n\left(n^{2}+1\right) \mathfrak{d}^{(4)}{ }_{(i j k l)}-2\left(n^{2}-4\right) \delta_{(i j} \delta_{k l)}, \\
d_{i j k l m} & \sim n\left(n^{2}+5\right) \mathfrak{d}^{(5)}{ }_{(i j k l m)}-2\left(3 n^{2}-20\right) \mathfrak{d}_{(i j k} \delta_{l m)}, \cdots
\end{aligned}
$$

up to numerical coefficients dependent on $n$. The $d$-tensors vanish when their order is larger than $n$. The $d$-tensors are totally symmetric and are orthogonal to all other $d$-tensors of different order. For instance, for the fourth-order tensor this means

$$
d_{i j k l} \delta_{i j}=0, \quad d_{i j k l} d_{i j k}=0 .
$$

Thus, the maximal contraction of the indices of two $d$-tensors of different order is zero. Using trace formulas for $\mathfrak{d}$-tensors

$$
\mathfrak{d}_{(i j k l)} \mathfrak{d}_{i j m}=\frac{2}{3} \frac{\left(n^{2}-8\right)}{n} \mathfrak{d}_{k l m}
$$

we can find the contraction of two indices for the third-order and fourth-order $d$-tensor

$$
d_{i j k l} d_{i j m} \sim d_{k l m} .
$$

Combinations of $d$-tensors provide a basis for the vector space of symmetric invariant polynomials of $\mathrm{SU}(n)$.

For $N=3$, the tensor $d_{a b c}$ takes the following values

$$
\begin{aligned}
& d_{118}=\frac{1}{\sqrt{3}} \quad d_{228}=\frac{1}{\sqrt{3}} \quad d_{338}=\frac{1}{\sqrt{3}} \quad d_{888}=\frac{-1}{\sqrt{3}} \\
& d_{448}=\frac{-1}{2 \sqrt{3}} \quad d_{558}=\frac{-1}{2 \sqrt{3}} \quad d_{668}=\frac{-1}{2 \sqrt{3}} \quad d_{778}=\frac{-1}{2 \sqrt{3}} \\
& d_{146}=\frac{1}{2} \quad d_{157}=\frac{1}{2} \quad d_{247}=-\frac{1}{2} \quad d_{256}=\frac{1}{2} \\
& d_{344}=\frac{1}{2} \quad d_{355}=\frac{1}{2} \quad d_{366}=-\frac{1}{2} \quad d_{377}=-\frac{1}{2}
\end{aligned}
$$


The anti-symmetric tensor $f_{a b c}$ takes the following values

$$
\begin{aligned}
& f_{123}=1 \quad f_{147}=\frac{1}{2} \quad f_{156}=-\frac{1}{2} \\
& f_{246}=\frac{1}{2} \quad f_{257}=\frac{1}{2} \quad f_{345}=\frac{1}{2} \\
& f_{367}=-\frac{1}{2} \quad f_{458}=\frac{\sqrt{3}}{2} \quad f_{678}=\frac{\sqrt{3}}{2}
\end{aligned}
$$

\section{B Primary fields}

Here, we write down the full primary operators corresponding to the quasi-primary operators in section 3.2.1 and section 3.2.2. Note that all fields are defined only up to an overall normalization factor.

$\boldsymbol{N}=\mathbf{2}$ primaries. The primary field of eq. (3.27) is

$$
\begin{aligned}
P_{6,1}= & \left(: \partial^{3} J^{a} \partial J^{a}:-: \partial^{2} J^{a} \partial^{2} J^{a}:-\frac{1}{10}: \partial^{4} J^{a} J^{a}:\right)+\frac{15288}{16465}: \partial^{2} T T:+\frac{5838}{16465}: \partial T \partial T: \\
& +\frac{653}{16465} \partial^{4} T-\frac{56}{135} \partial^{2} Q_{4}+\frac{112}{45}: Q_{4} T:-\frac{22176}{16465}: T T T: .
\end{aligned}
$$

$\boldsymbol{N}=\mathbf{3}$ primaries. The primary completion of the quasi-primary field $Q_{4}$ is

$$
P_{4}=Q_{4}-\frac{9}{31} \partial^{2} T+\frac{30}{31}: T T: .
$$

The spin 5 primary field is

$$
P_{5}=Q_{5}+\frac{6}{17} \partial^{2} Q_{3}-\frac{42}{85}: Q_{3} T: .
$$

The bilinear spin 6 primary is

$$
\begin{aligned}
P_{6,1}= & Q_{6,1}-\frac{231}{620}: \partial^{2} T T:-\frac{21}{310}: \partial T \partial T:+\frac{21}{20}: Q_{4} T: \\
& +\frac{79}{155} \partial^{4} T-\frac{49}{40} \partial^{2} Q_{4}+\frac{147}{310}: T T T: .
\end{aligned}
$$

The trilinear spin 6 primary is

$$
P_{6,2}=Q_{6,2}-\frac{3}{5} \partial^{3} P_{3}+\frac{24}{5}: \partial P_{3} T:-\frac{36}{5}: \partial T P_{3}:
$$

\section{Algebra of symmetric product orbifold CFT}

The most straightforward way to find the spin and multiplicity of the generators of the symmetry algebra for the symmetric product orbifold theory is by looking at its vacuum character. Let us denote the chiral vacuum character of a seed theory by

$$
\chi_{1}(q)=\sum_{m=0}^{\infty} a_{m} q^{m} .
$$


Then the vacuum character of the $N^{\prime}$ th symmetric product orbifold can be read off from the following plethystic exponential [60-62]

$$
\chi(\nu, q)=\prod_{m=0}^{\infty} \frac{1}{\left(1-\nu q^{m}\right)^{a_{m}}}=\exp \left(\sum_{k=0}^{\infty} \frac{1}{k} \chi_{1}\left(q^{k}\right) \nu^{k}\right) .
$$

Expanding the exponential in the r.h.s. gives a series in powers of $\nu$, we get:

$$
\begin{aligned}
\chi(\nu, q) & =\sum_{N=0}^{\infty} \chi_{N}(q) \nu^{N} \\
& =1+\chi_{1}(q) \nu+\frac{\chi_{1}(q)^{2}+\chi_{1}\left(q^{2}\right)}{2} \nu^{2}+\frac{\chi_{1}(q)^{3}+3 \chi_{1}(q) \chi_{1}\left(q^{2}\right)+2 \chi_{1}\left(q^{3}\right)}{6} \nu^{3}+\cdots
\end{aligned}
$$

We can find the vacuum character for the $N$ 'th symmetric orbifold CFT by reading off the coefficients of $\nu^{N}$. In our case, the seed theory is the single boson theory whose chiral character is given by

$$
\chi_{1}(q)=\sum_{m=0}^{\infty} a_{m} q^{m}=\prod_{n=1}^{\infty} \frac{1}{\left(1-q^{n}\right)} .
$$

Using this expression for $\chi_{1}$, we can compute the the characters and the corresponding symmetry algebra of the symmetric product orbifold CFT using eq. (C.3). These chiral characters agree with standard results for $S_{N}$ orbifolds [63, 64]. From these characters, we can compute the spectrum of the algebra for small values of $N$ :

$$
\begin{array}{ll}
N=2: & 1,2,4 . \\
N=3: & 1,2,3,4,5,6^{2} . \\
N=4: & 1,2,3,4^{2}, 5,6^{3}, 7^{2}, 8^{3}, 9 . \\
N=5: & 1,2,3,4^{2}, 5^{2}, 6^{3}, 7^{3}, 8^{5}, 9^{4}, 10^{5}, 11 . \\
N=6: & 1,2,3,4^{2}, 5^{2}, 6^{4}, 7^{3}, 8^{6}, 9^{6}, 10^{8}, 11^{7}, 12^{8}, 13 .
\end{array}
$$

As for the coset case, there can be more generators present. Despite the initial exponential growth in the number of operators with spin, at finite $N$, null states start appearing is the spectrum at some finite value of the spin and thus the algebra truncates. This is reflected in the asymptotic growth of the vacuum character:

$$
n^{-\frac{3}{4}} \exp \left(\pi \sqrt{\frac{2}{3} n N}\right) \text {. }
$$

which exihibits Cardy growth as $n \rightarrow \infty$.

As $N \rightarrow \infty$, the vacuum character is given by

$$
\lim _{\nu \rightarrow 1}(1-\nu) \chi(\nu, q)=\prod_{m=0}^{\infty} \frac{1}{\left(1-q^{m}\right)^{a_{m}}},
$$

which is again the plethystic exponential of eq. (C.4). This can be rewritten as

$$
\prod_{n=1}^{\infty} \frac{1}{\left(1-q^{n}\right)^{a_{1}}} \prod_{m=2}^{\infty} \prod_{n=m}^{\infty} \frac{1}{\left(1-q^{n}\right)^{a_{m}-a_{m-1}}} .
$$




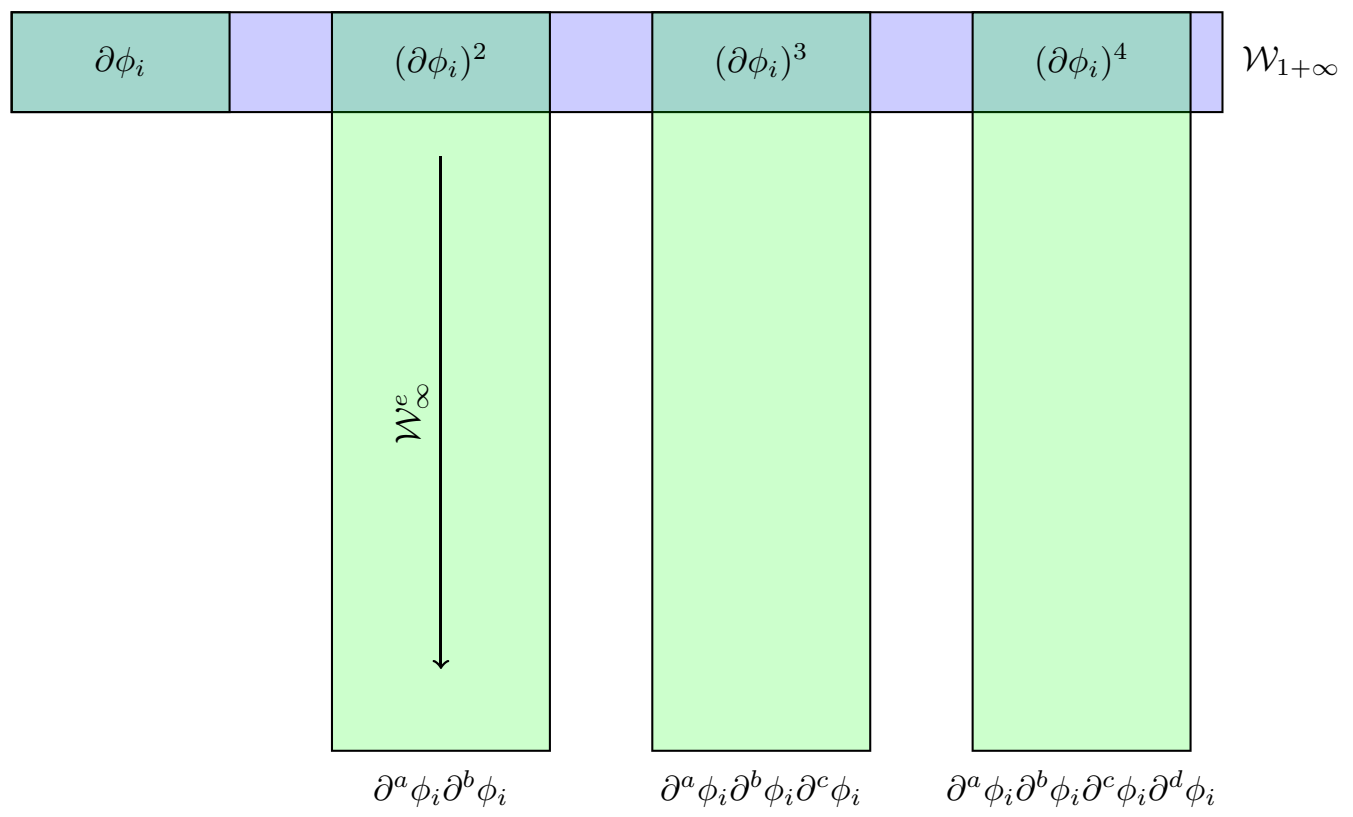

Figure 2. The higher spin square is generated by acting by derivatives on the operators in the top row. The second column corresponds to the $\mathcal{W}_{\infty}^{e}[1]$ algebra while subsequent columns correspond to its representations.

The generators of the infinite $N$ algebra are thus enumerated by the generating function

$$
\prod_{n=2}^{\infty} \frac{1}{\left(1-q^{n}\right)}
$$

along with a spin one field.

We now write down the exact form of the currents. In the large $N$ limit, the "single-particle" generators for the symmetric product orbifold are symmetrized products of the form

$$
\sum_{i=1}^{N}\left(\partial^{m_{1}} \phi_{i}\right) \cdots\left(\partial^{m_{p}} \phi_{i}\right), \quad m_{1}, \ldots, m_{p} \geq 1 .
$$

Because of the symmetrization over $N$, this set of generators is in one-to-one correspondence with the chiral sector of a single boson. Removing the terms that are total derivatives, and in the $N \rightarrow \infty$ limit, they also constitute a set of linearly independent operators. Out of these generators, the subset of generators of eq. (C.9) of the form

$$
\sum_{i=1}^{N}\left(\partial^{m_{1}} \phi_{i}\right)\left(\partial^{m_{2}} \phi_{i}\right), \quad m_{1}, m_{2} \geq 1,
$$

define quasiprimary generators of spin $s=m_{1}+m_{2}$, in specific linear combinations and when $s$ is even. In fact, only one independent current can be constructed at each even spin, meaning that it is not a linear combination of derivatives of lower-spin currents, and there are no independent odd-spin currents. This set of independent currents generate 
the even spin $\mathcal{W}$-algebra $\mathcal{W}_{\infty}^{e}[1]$. The generators in eq. (C.10) are of order two, i.e., they are bilinear in the $\phi \mathrm{s}$. The currents in eq. (C.9) are of arbitrary order $p \geq 1$. However, it turns out that the currents of a fixed order $p$, suitably corrected by lower-order terms, form a representation of the wedge algebra of $\mathcal{W}_{\infty}^{e}[1]$. This is captured in figure (2) where currents of a given order correspond to columns. The operators of the symmetric product orbifold algebra are, therefore, organized into representations of $\mathcal{W}_{\infty}^{e}[1]$. The statement that operators of the higher spin square can be organized in representations of $\mathcal{W}_{\infty}^{e}[1]$ is captured by the following identity:

$$
\prod_{n=1}^{\infty} \frac{1}{\left(1-q^{n}\right)}=1+\sum_{p=1}^{\infty} b_{\left(\left[0^{p-1}, 1,0, \ldots, 0\right] ; 0\right)}^{(\text {wedge }[\lambda=1]}
$$

where $b_{\left(\left[0^{p-1}, 1,0, \ldots, 0\right] ; 0\right)}^{(\text {wedge }[\lambda=1]}$ denotes the wedge character of the $\left(\left[0^{p-1}, 1,0, \ldots, 0\right] ; 0\right)$ representation of $\mathcal{W}_{\infty}^{e}[1]$. The $b_{\left(\left[0^{p-1}, 1,0, \ldots, 0\right] ; 0\right)}^{(\text {wedg }[\lambda=1]}$ character is defined in eq. (3.57).

The l.h.s. of eq. (C.11) is the normalized partition function for a single boson. Combinatorially, the l.h.s. is just the generating function for the number of ways one can partition an integer varying from 1 to $\infty$ into an arbitrary number of parts. Each term in the sum on the r.h.s. is number of ways one can partition an integer into exactly $p$ parts. In terms of the operators in eq. (C.9), this is the spin $s$ of the operator, varying from $p$ to $\infty$, being partitioned into $m_{1}, m_{2}, \ldots, m_{p}$ at fixed $p$. An alternate way to organize the operators of the higher spin square is in terms of representations of $\mathcal{W}_{1+\infty}[0]$. For more details, see $[36,65]$.

Open Access. This article is distributed under the terms of the Creative Commons Attribution License (CC-BY 4.0), which permits any use, distribution and reproduction in any medium, provided the original author(s) and source are credited.

\section{References}

[1] M.R. Gaberdiel and R. Gopakumar, Minimal model holography, J. Phys. A 46 (2013) 214002 [arXiv: 1207.6697 ] [INSPIRE].

[2] M.R. Gaberdiel and R. Gopakumar, An AdS 3 dual for minimal model CFTs, Phys. Rev. D 83 (2011) 066007 [arXiv: 1011.2986] [INSPIRE].

[3] M.R. Gaberdiel, R. Gopakumar and A. Saha, Quantum W-symmetry in AdS $S_{3}$, JHEP 02 (2011) 004 [arXiv:1009.6087] [INSPIRE].

[4] E.S. Fradkin and M.A. Vasiliev, Candidate to the role of higher spin symmetry, Annals Phys. 177 (1987) 63 [INSPIRE].

[5] M.P. Blencowe, A consistent interacting massless higher spin field theory in $D=(2+1)$, Class. Quant. Grav. 6 (1989) 443 [InSPIRE].

[6] S.F. Prokushkin and M.A. Vasiliev, Higher spin gauge interactions for massive matter fields in 3 - D AdS space-time, Nucl. Phys. B 545 (1999) 385 [hep-th/9806236] [InSPIRE].

[7] M. Henneaux and S.-J. Rey, Nonlinear $W_{\infty}$ as asymptotic symmetry of three-dimensional higher spin Anti-de Sitter gravity, JHEP 12 (2010) 007 [arXiv: 1008.4579] [INSPIRE]. 
[8] A. Campoleoni, S. Fredenhagen, S. Pfenninger and S. Theisen, Asymptotic symmetries of three-dimensional gravity coupled to higher-spin fields, JHEP 11 (2010) 007 [arXiv: 1008.4744] [INSPIRE].

[9] A. Campoleoni, S. Fredenhagen and S. Pfenninger, Asymptotic W-symmetries in three-dimensional higher-spin gauge theories, JHEP 09 (2011) 113 [arXiv:1107.0290] [INSPIRE].

[10] T. Creutzig, Y. Hikida and P.B. Ronne, Extended higher spin holography and Grassmannian models, JHEP 11 (2013) 038 [arXiv: 1306. 0466] [INSPIRE].

[11] C. Candu and C. Vollenweider, On the coset duals of extended higher spin theories, JHEP 04 (2014) 145 [arXiv: 1312.5240] [INSPIRE].

[12] A.B. Zamolodchikov, Infinite additional symmetries in two-dimensional conformal quantum field theory, Theor. Math. Phys. 65 (1985) 1205 [Teor. Mat. Fiz. 65 (1985) 347] [INSPIRE].

[13] I. Bakas and E. Kiritsis, Bosonic realization of a universal $W$-algebra and $Z_{\infty}$ parafermions, Nucl. Phys. B 343 (1990) 185 [Erratum ibid. B 350 (1991) 512] [InSPIRE].

[14] M.R. Gaberdiel and R. Gopakumar, Triality in minimal model holography, JHEP 07 (2012) 127 [arXiv: 1205.2472] [INSPIRE].

[15] A.R. Linshaw, Universal two-parameter $\mathcal{W}_{\infty}$-algebra and vertex algebras of type $\mathcal{W}(2,3, \ldots, N)$, arXiv: 1710.02275 [INSPIRE].

[16] P. Bouwknegt and K. Schoutens, $W$ symmetry in conformal field theory, Phys. Rept. 223 (1993) 183 [hep-th/9210010] [inSPIRE].

[17] F.A. Bais, P. Bouwknegt, M. Surridge and K. Schoutens, Extensions of the Virasoro algebra constructed from Kac-Moody algebras using higher order Casimir invariants, Nucl. Phys. B 304 (1988) 348 [INSPIRE].

[18] F.A. Bais, P. Bouwknegt, M. Surridge and K. Schoutens, Coset construction for extended Virasoro algebras, Nucl. Phys. B 304 (1988) 371 [INSPIRE].

[19] J. de Boer, L. Feher and A. Honecker, A class of $W$ algebras with infinitely generated classical limit, Nucl. Phys. B 420 (1994) 409 [hep-th/9312049] [INSPIRE].

[20] R. Gopakumar et al., Strange metals in one spatial dimension, Phys. Rev. D 86 (2012) 066003 [arXiv: 1206.4719] [INSPIRE].

[21] C.-h. Ahn, K. Schoutens and A. Sevrin, The full structure of the super W(3) algebra, Int. J. Mod. Phys. A 6 (1991) 3467 [INSPIRE].

[22] C. Ahn, The higher spin currents in the $N=1$ stringy coset minimal model, JHEP 04 (2013) 033 [arXiv: 1211.2589] [INSPIRE].

[23] C. Ahn, Higher spin currents in the $N=2$ stringy coset minimal model, Phys. Rev. D 94 (2016) 126014 [arXiv: 1604.00756] [INSPIRE].

[24] C. Ahn, Higher spin currents with arbitrary $N$ in the $\mathcal{N}=\infty$ stringy coset minimal model, JHEP 07 (2013) 141 [arXiv: 1305.5892] [INSPIRE].

[25] P. Bowcock and P. Goddard, Coset constructions and extended conformal algebras, Nucl. Phys. B 305 (1988) 685 [INSPIRE].

[26] V.G. Kac and D.H. Peterson, Infinite dimensional Lie algebras, theta functions and modular forms, Adv. Math. 53 (1984) 125 [INSPIRE]. 
[27] A. Nazarov, Affine.m - Mathematica package for computations in representation theory of finite-dimensional and affine Lie algebras, Comput. Phys. Commun. 183 (2012) 2480 [arXiv: 1107.4681] [INSPIRE].

[28] R. Blumenhagen, $W$ algebras in conformal quantum theory, BONN-IR-91-06 (1991).

[29] V.G. Kac and M. Wakimoto, Modular and conformal invariance constraints in representation theory of affine algebras, Adv. Math. 70 (1988) 156 [INSPIRE].

[30] C. Ahn, The coset spin-4 Casimir operator and its three-point functions with scalars, JHEP 02 (2012) 027 [arXiv: 1111.0091] [INSPIRE].

[31] C. Ahn and H. Kim, Spin-5 Casimir operator its three-point functions with two scalars, JHEP 01 (2014) 012 [Erratum ibid. 01 (2014) 174] [arXiv:1308.1726] [INSPIRE].

[32] M.R. Gaberdiel and C. Vollenweider, Minimal model holography for $\mathrm{SO}(2 \mathrm{~N})$, JHEP 08 (2011) 104 [arXiv: 1106.2634] [INSPIRE].

[33] C. Candu, M.R. Gaberdiel, M. Kelm and C. Vollenweider, Even spin minimal model holography, JHEP 01 (2013) 185 [arXiv:1211.3113] [INSPIRE].

[34] C. Candu and C. Vollenweider, The $\mathcal{N}=1$ algebra $\mathcal{W}_{\infty}[\mu]$ and its truncations, JHEP 11 (2013) 032 [arXiv: 1305.0013] [inSPIRE].

[35] D. Kumar and M. Sharma, Conformal embeddings and higher-spin bulk duals, Phys. Rev. D 95 (2017) 066015 [arXiv: 1606.00791] [INSPIRE].

[36] M.R. Gaberdiel and R. Gopakumar, Stringy symmetries and the higher spin square, J. Phys. A 48 (2015) 185402 [arXiv:1501.07236] [INSPIRE].

[37] K. Thielemans, A Mathematica package for computing operator product expansions, Int. J. Mod. Phys. C 2 (1991) 787 [INSPIRE].

[38] G.E. Andrews, The theory of partitions, Encyclopedia of Mathematics and its Applications, Cambridge University Press, Cambridge U.K. (1998).

[39] J.A. de Azcarraga, A.J. Macfarlane, A.J. Mountain and J.C. Perez Bueno, Invariant tensors for simple groups, Nucl. Phys. B 510 (1998) 657 [physics/9706006] [INSPIRE].

[40] J.A. de Azcarraga and A.J. Macfarlane, Compilation of relations for the antisymmetric tensors defined by the Lie algebra cocycles of SU(N), Int. J. Mod. Phys. A 16 (2001) 1377 [math-ph/0006026] [INSPIRE].

[41] D. Gepner, New conformal field theories associated with Lie algebras and their partition functions, Nucl. Phys. B 290 (1987) 10 [INSPIRE].

[42] T. Procházka, Exploring $\mathcal{W}_{\infty}$ in the quadratic basis, JHEP 09 (2015) 116 [arXiv:1411.7697] [INSPIRE].

[43] P. Dittner, Invariant tensors in SU(3). 2., Commun. Math. Phys. 27 (1972) 44 [InSPIRE].

[44] P. Dittner, Invariant tensors in SU(3), Commun. Math. Phys. 22 (1971) 238 [inSPIRE].

[45] R. Blumenhagen et al., Coset realization of unifying $W$ algebras, Int. J. Mod. Phys. A 10 (1995) 2367 [hep-th/9406203] [INSPIRE].

[46] R. Blumenhagen et al., Unifying W algebras, Phys. Lett. B 332 (1994) 51 [hep-th/9404113] [INSPIRE].

[47] M.R. Gaberdiel, R. Gopakumar, T. Hartman and S. Raju, Partition functions of holographic minimal models, JHEP 08 (2011) 077 [arXiv: 1106.1897] [INSPIRE]. 
[48] C.N. Pope, Lectures on $W$ algebras and $W$ gravity, hep-th/9112076 [INSPIRE].

[49] B. Sundborg, Stringy gravity, interacting tensionless strings and massless higher spins, Nucl. Phys. Proc. Suppl. 102 (2001) 113 [hep-th/0103247] [INSPIRE].

[50] P. Haggi-Mani and B. Sundborg, Free large $N$ supersymmetric Yang-Mills theory as a string theory, JHEP 04 (2000) 031 [hep-th/0002189] [INSPIRE].

[51] L. Eberhardt, M.R. Gaberdiel and R. Gopakumar, The worldsheet dual of the symmetric product CFT, JHEP 04 (2019) 103 [arXiv:1812.01007] [INSPIRE].

[52] M.R. Gaberdiel and R. Gopakumar, Tensionless string spectra on $A d S_{3}$, JHEP 05 (2018) 085 [arXiv: 1803. 04423] [INSPIRE].

[53] G. Giribet et al., Superstrings on $A d S_{3}$ at $\|=1$, JHEP 08 (2018) 204 [arXiv:1803.04420] [INSPIRE].

[54] M.R. Gaberdiel, R. Gopakumar and C. Hull, Stringy AdS $S_{3}$ from the worldsheet, JHEP 07 (2017) 090 [arXiv : 1704.08665] [INSPIRE].

[55] O. Ohlsson Sax and B. Stefański, Closed strings and moduli in $A d S_{3} / C F T_{2}$, JHEP 05 (2018) 101 [arXiv: 1804.02023] [INSPIRE].

[56] O. Ohlsson Sax, A. Sfondrini and B. Stefanski, Integrability and the conformal field theory of the Higgs branch, JHEP 06 (2015) 103 [arXiv:1411.3676] [INSPIRE].

[57] M. Baggio et al., Protected string spectrum in $A d S_{3} / C F T_{2}$ from worldsheet integrability, JHEP 04 (2017) 091 [arXiv: 1701.03501] [INSPIRE].

[58] T. Creutzig and Y. Hikida, Rectangular W-algebras, extended higher spin gravity and dual coset CFTs, JHEP 02 (2019) 147 [arXiv:1812.07149] [INSPIRE].

[59] A. Sudbery, Computer-friendly d-tensor identities for SU(n), J. Phys.A 2315 (1990) L705.

[60] R. Dijkgraaf, G.W. Moore, E.P. Verlinde and H.L. Verlinde, Elliptic genera of symmetric products and second quantized strings, Commun. Math. Phys. 185 (1997) 197 [hep-th/9608096] [INSPIRE].

[61] S. Benvenuti, B. Feng, A. Hanany and Y.-H. He, Counting BPS operators in gauge theories: quivers, syzygies and plethystics, JHEP 11 (2007) 050 [hep-th/0608050] [INSPIRE].

[62] B. Feng, A. Hanany and Y.-H. He, Counting gauge invariants: the plethystic program, JHEP 03 (2007) 090 [hep-th/0701063] [INSPIRE].

[63] P. Bantay, Permutation orbifolds, Nucl. Phys. B 633 (2002) 365 [hep-th/9910079] [INSPIRE].

[64] A. Jevicki and J. Yoon, $S_{N}$ orbifolds and string interactions, J. Phys. A 49 (2016) 205401 [arXiv: 1511.07878] [INSPIRE].

[65] M. Sharma, The higher spin rectangle, JHEP 01 (2018) 073 [arXiv:1708.04996] [INSPIRE]. 